\title{
An experimental study on thermal circulation driven by horizontal differential heating
}

\author{
By WEI WANG ${ }^{1}$ AND RUI XIN HUANG \\ ${ }^{1}$ Physical Oceanography Lab, Ocean University of China, Qingdao 266003, \\ Shandong, PR China \\ ${ }^{2}$ Department of Physical Oceanography, Woods Hole Oceanographic Institution, \\ Woods Hole, MA 02543, USA
}

(Received 5 April 2004 and in revised form 29 March 2005)

Circulation driven by horizontal differential heating is studied, using a doublewalled Plexiglas tank $\left(20 \times 15 \times 2.5 \mathrm{~cm}^{3}\right)$ filled with salt water. For instances of heating/cooling from above and below, results indicate that there is always quasi-equilibrium circulation. In contrast to most previous results from experimental/numerical studies, circulation in our experiments appears in the form of a shallow cell adjacent to the boundary of thermal forcing. The non-dimensional streamfunction maximum confirms the 1/5-power law of Rossby, $\Psi \sim R a_{L}^{1 / 5}$. Dissipation rate measured in the experiments appears to be consistent with theory.

For cases of heating/cooling from a sloping bottom, circulation is similar to cases with a flat bottom; circulation is strong if heating is below cooling, but it is rather weak if heating is above cooling. Nevertheless, circulation in all cases is visible to the naked eye.

\section{Introduction}

Traditional theory of oceanic circulation is based on the assumption that the meridional overturning circulation is driven by the surface buoyancy forcing. However, a new paradigm is emerging, in which external mechanical energy plays an important role in driving the meridional overturning circulation (e.g. Munk \& Wunsch 1988; Huang 1999, 2004; Wunsch \& Ferrari 2004). As a mechanical system, oceanic circulation requires sources of mechanical energy to overcome friction. There are external sources of mechanical energy in the oceans, including wind stress and tidal dissipation. In addition, geothermal heating can also generate a small amount of mechanical energy supporting the circulation.

It is generally accepted that surface thermal forcing is also important in regulating the meridional circulation in the oceans; however, the exact role of surface thermal forcing remains debatable. An important question is whether surface thermal forcing alone can drive circulation in the oceans. A continuing debate exists over this issue, starting with the classical hypothesis of Sandström $(1908,1916)$. Note that his arguments were based on thermodynamics rather than fluid dynamical analysis. Over the past 100 years, his postulations have evolved, and are frequently referred to as 'the Sandström theorem'. His original papers in German are not easily accessible, but a concise statement of the Sandström theorem can be found in Defant (1961, p. 491): a closed steady circulation can only be maintained in the ocean if the heat source is situated at a lower level than the cold source. 
A close examination reveals that circulation driven solely by thermal forcing can be classified into the following three types.

Type 1. The heating source is placed at a pressure level higher than the cooling source; it is well known that there is strong circulation.

Type 2. The heating source is placed at a pressure level lower than the cooling source. Sandström (1908) carried out such a laboratory experiment and reported that there was no circulation in the final steady state (see Defant 1961, p. 491). However, Jeffreys (1925) argued that wherever there is a horizontal density difference, there should be circulation; but he did not state how fast the circulation would be. Although Type 2 thermal circulation can be weak, the circulation is detectable. Miller (1968) carried out a Type 2 experiment and noted weak circulation, consisting of clockwise motion in the upper half of the tank and anticlockwise motion in the lower half of the tank, which can be inferred from the streak photograph of aluminium particles released in the fluid. However, no quantitative data were included in his thesis.

Type 3. Heating and cooling sources placed at the same pressure level (also referred to as horizontal differential heating or horizontal convection). This type of heating/cooling resembles situations in the ocean which is primarily heated and cooled from the upper surface, neglecting the penetration of solar radiation and geothermal heating. The long-standing question is whether thermal forcing alone in such a setting can drive a circulation. Rossby (1965) carried out a series of laboratory experiments in thermal circulation under horizontal differential heating, and reported steady circulation that occupied the entire depth of the tank. Results from numerical experiments by Rossby $(1965,1998)$ are consistent with his laboratory experiments. Results from a recent experimental study of imposed heat flux (Mullarney, Griffiths \& Hughes 2004) also fit the 1/5-power law of Rossby (1965). The tank used in their experiments is larger than that of Rossby, and they described the flows as turbulent. However, according to a more strict definition of turbulence, such flows are nonturbulent (Paparella \& Young 2002).

Numerical experiments have been discussed by other investigators (e.g. Somerville 1967; Beardsley \& Festa 1973; Paparella \& Young 2002; Siggers, Kerswell \& Balmforth 2004). Most of these numerical solutions indicate circulation occupying the entire depth of the tank. Numerical solutions of Paparella \& Young (2002), however, showed that as the Prandtl number increases to 10 , the circulation has a tendency to appear in forms of a shallow cell. If we could extrapolate these results to the oceans, then the deep circulation can be driven by surface thermal forcing alone.

According to the formulae of Paparella \& Young (2002), the amount of mechanical energy convertible from surface thermal forcing in the world oceans is about $1.5 \mathrm{GW}$ (see the Appendix). On the other hand, the world oceans receive a large amount of external mechanical energy, of the order of $64 \mathrm{TW}$, from wind stress and tidal dissipation (Huang 2004); thus, the meridional overturning circulation in the world oceans, at least the way they exist on the Earth, should be classified as a conveyer machine driven by external sources of mechanical energy (Wunsch \& Ferrari 2004). Nevertheless, theoretically, the ocean under surface thermal forcing alone can develop circulation: even though such circulation may be very sluggish, the ocean can function as a heat engine in such an idealized situation. In conclusion, although the socalled Sandström theorem is seemingly a sound hypothesis based on thermodynamic analysis, it is inaccurate, as discussed by Jeffreys (1925), Rossby (1965) and other investigators.

Our goal is to examine the circulation driven by horizontal convection in laboratory experiments. Rossby's experiments are now considered classical results. However, they 


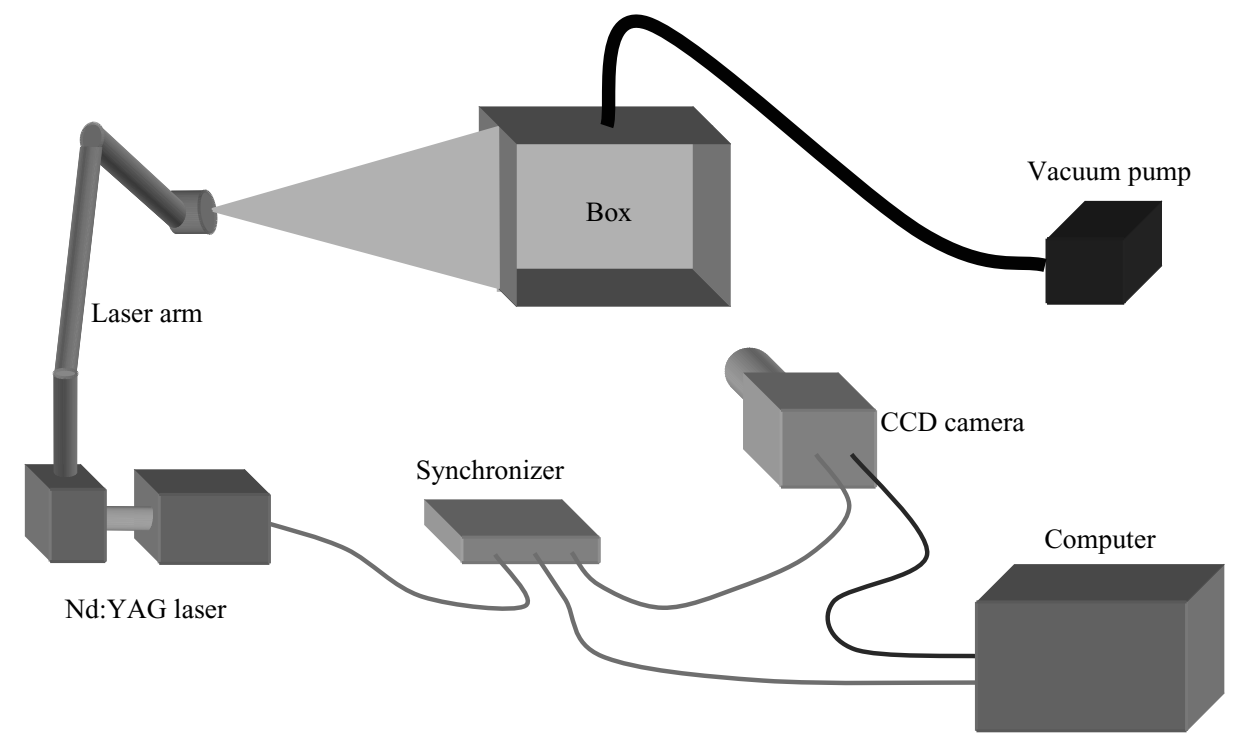

FIGURE 1. Schematic of the experimental set-up.

were performed nearly 40 years ago and several aspects of Rossby's experiments seem improvable with the aid of modern technology. (i) Rossby's experiments were performed with an open slot on the top where temperature measurements were carried out. (ii) The temperature of the heating source was higher than the environment. Both facts may compromise the thermal isolation of the tank. (iii) Most of his experiments lasted less than $10 \mathrm{~h}$, so the system may not have had enough time to reach final equilibrium; and (iv) The velocity and circulation patterns were inferred from photographs of tracers, so velocity measurements were not very accurate.

With advances in technology and renewed interest in understanding the physics, it is time to re-examine these issues, but under more accurate conditions. This paper is organized as follows: the experimental equipment and techniques used are described in $§ 2$. This study has been focused on velocity measurements obtained from newly available laser image analysis technology. With these new techniques, we can measure velocity with unprecedented accuracy. Results of these measurements are discussed in $\S 3$, with the scaling of circulation discussed in the Appendix. Conclusions are drawn in $\S 4$.

\section{Experimental set-up}

A sketch of the experimental set-up is shown in figure 1. The experiments were carried out in a Plexiglas tank with internal dimensions of $20 \mathrm{~cm} \times 15 \mathrm{~cm} \times 2.5 \mathrm{~cm}$. In order to cut down possible heat exchange within the external environment, the tank was double-walled with a gap of $1 \mathrm{~cm}$ between the inner and outer Plexiglas walls, which were $2 \mathrm{~cm}$ thick in order to sustain the pressure difference. A high vacuum of $2 \times 10^{-7}$ bar was maintained during all experiments.

The base of the inner box was made of copper $1 \mathrm{~cm}$ thick and covered by Plexiglas $2 \mathrm{~cm}$ thick to act as insulation from the external environment. A steady temperature distribution was maintained in the copper by heating one end and cooling at the other end with a pair of thermoelectric coolers (TEC). Since thick copper bar is a 
better heat conductor than water, the fluid in the tank is considered forced by a linear temperature profile on the bottom (or upper) boundary. The maximum output power of TEC is $30 \mathrm{~W}$ and controlled by a RKC digital controller; the instrument maintains temperature to the target value with an accuracy of $\pm 0.1 \mathrm{~K}$. For a dry test, i.e. the instrument is in touch with air and without water, it takes less than 3 min to reach the target temperature. In our experiments, it typically takes about 30 min to reach the target temperature within accuracy. Initial temperature of the fluid is measured by a thermocouple embedded in the copper. The room temperature is carefully controlled to a desired level.

For each experiment, a filled water tank sat for at least $6 \mathrm{~h}$ to guarantee a nearly thermal equilibrium between the fluid in the tank and the environment. The vacuum pump was then turned on; it took 4 to $5 \mathrm{~h}$ to achieve the high vacuum desired. During this period, the process of achieving equilibrium between the fluid and the environment continued. Afterward, heating/cooling sources were switched on, and the majority of experiments were run for $10-80 \mathrm{~h}$ to ensure that circulation reached a quasi-equilibrium state, i.e. the temporal mean velocity field of each measurement becomes steady.

Although the vacuum of double walls reduces heat exchange within the environment, radiation can be another non-negligible source of heat exchange. In order to maximally reduce the potential heat exchange within the environment, the room temperature and the temperature of the heating/cooling sources are carefully chosen and maintained (table 1). Note that salt concentration used in this study is far beyond the range where a conventional equation of state is defined, so the density difference of salt water is calculated using data from Lide (2001).

For instances of heating/cooling from below, the temperature of the heating source is set to slightly below the initial temperature of the water (except for run 10). In the final quasi-equilibrium state, water above the circulation cell in the tank has a temperature slightly lower than the heating source. Thus, it is slightly colder than the environment, and the weak heat exchange due to background radiation imposes heating from above. To balance the downward heat flux, cooling must take place through the lower boundary in compensation. Therefore, radiation-induced heat leakage is equivalent to heating from above and cooling from below, a stabilized situation implying that errors in the velocity field owing to heat leakage should be minimal.

Similarly, for cases of heating/cooling from above, the initial temperature is chosen to be slightly lower than the cooling source. Thus, in the final quasi-equilibrium state, heat leakage due to radiation is cooling from below and heating from above, and the errors due to heat leakage are minimal.

Our setting is similar to that of Miller (1968). A concise description of Miller's experiments is as follows. The fluid tank is a box $(12.7 \mathrm{~cm} \times 6.1 \mathrm{~cm})$ made of Plexiglas of $1.3 \mathrm{~cm}$ thick and it is enclosed in a vacuum chamber made of Plexiglas of $2.54 \mathrm{~cm}$ thick. The base of the inner box is formed by an aluminium bar with heating in the middle and cooling at both ends; thus, temperature increased linearly from left to centre and decreased linearly from centre to the right. The fluid used in the experiments was a silicone liquid Dow Corning 200. Velocity measurements were obtained by introducing suspended fine aluminium particles into the fluid and photographing their movement by time exposure with a camera. Temperature distribution in the bottom boundary layer was measured by a thermocouple probe, performed without the use of vacuum insulation. A modified schlieren system was used to study the vertical gradient of temperature within the fluid. The experiments 


\begin{tabular}{|c|c|c|c|c|c|c|c|c|c|}
\hline $\begin{array}{c}\text { Run } \\
\text { number }\end{array}$ & Case & $T_{e}$ & $T_{h}$ & $T_{c}$ & Salinity & $g^{\prime}$ & $v \times 10^{2}$ & $\kappa \times 10^{3}$ & Duration (h) \\
\hline 1 & 1 & 23.3 & 22.5 & 4.0 & 0.25 & 2.70 & 1.21 & 1.41 & 12 \\
\hline 2 & 1 & 23.1 & 22.5 & 4.0 & 0.25 & 2.70 & 1.21 & 1.41 & 10 \\
\hline 3 & 1 & 23.7 & 22.5 & 4.0 & 3.0 & 6.50 & 1.37 & 1.47 & 26 \\
\hline 4 & 1 & 31.2 & 30.0 & 2.0 & 5.0 & 11.33 & 1.38 & 1.50 & 9 \\
\hline 5 & 1 & 30.6 & 27.0 & 12.0 & 5.0 & 6.18 & 1.28 & 1.50 & 10 \\
\hline 6 & 1 & 30.8 & 27.0 & 2.0 & 5.0 & 10.04 & 1.42 & 1.50 & 10 \\
\hline 7 & 1 & 23.6 & 21.5 & 19.5 & 5.0 & 0.83 & 1.25 & 1.50 & 10 \\
\hline 8 & 1 & 24.5 & 23.0 & 18.0 & 5.0 & 2.06 & 1.25 & 1.50 & 10 \\
\hline 9 & 1 & 27.5 & 25.0 & 18.0 & 5.0 & 2.90 & 1.23 & 1.50 & 12 \\
\hline 10 & 1 & 23.8 & 25.0 & 23.0 & 5.0 & 0.84 & 1.17 & 1.51 & 42 \\
\hline 11 & 1 & 23.1 & 19.32 & 18.0 & 5.0 & 0.53 & 1.30 & 1.50 & 26 \\
\hline 12 & 1 & 23.4 & 21.0 & 19.47 & 5.0 & 0.63 & 1.26 & 1.50 & 26 \\
\hline 13 & 1 & 23.2 & 19.3 & 17.0 & 5.0 & 0.93 & 1.31 & 1.50 & 8 \\
\hline 14 & 1 & 23.0 & 20.0 & 17.0 & 5.0 & 1.21 & 1.30 & 1.50 & 8 \\
\hline 15 & 1 & 23.5 & 21.0 & 17.0 & 5.0 & 1.63 & 1.29 & 1.50 & 12 \\
\hline 16 & 1 & 23.3 & 21.0 & 16.0 & 5.0 & 2.03 & 1.30 & 1.50 & 10 \\
\hline 17 & 1 & 23.5 & 22.0 & 21.0 & 5.0 & 0.42 & 1.23 & 1.50 & 27 \\
\hline 18 & 1 & 23.1 & 22.5 & 22.0 & 5.0 & 0.21 & 1.21 & 1.50 & 13 \\
\hline 19 & 1 & 27.0 & 25.0 & 10.0 & 0.5 & 3.38 & 1.10 & 1.44 & 52 \\
\hline 20 & 1 & 20.0 & 22.0 & 10.0 & 0.5 & 2.57 & 1.14 & 1.43 & 80 \\
\hline 21 & 1 & 24.0 & 22.0 & 10.0 & 0.5 & 2.57 & 1.12 & 1.42 & 80 \\
\hline 22 & 2 & 15.8 & 22.0 & 17.0 & 5.0 & 2.05 & 1.28 & 1.50 & 12 \\
\hline 23 & 2 & 15.5 & 27.0 & 17.0 & 5.0 & 4.17 & 1.21 & 1.50 & 12 \\
\hline 24 & 2 & 16.3 & 35.5 & 17.0 & 5.0 & 7.91 & 1.12 & 1.51 & 15 \\
\hline 25 & 2 & 16.0 & 55.0 & 17.0 & 5.0 & 17.05 & 0.95 & 1.53 & 15 \\
\hline 26 & 3 & 23.3 & 22.5 & 4.0 & 3.0 & 6.50 & 1.37 & 1.47 & 20 \\
\hline 27 & 3 & 23.5 & 22.0 & 18.0 & 0.25 & 0.85 & 1.02 & 1.44 & 10 \\
\hline 28 & 3 & 23.5 & 22.0 & 15.0 & 0.25 & 1.38 & 1.06 & 1.44 & 11 \\
\hline 29 & 3 & 23.4 & 20.2 & 18.0 & 0.25 & 0.40 & 1.04 & 1.44 & 11 \\
\hline 30 & 4 & 23.7 & 22.5 & 4.0 & 3.0 & 6.50 & 1.37 & 1.47 & 20 \\
\hline 31 & 4 & 23.6 & 22.0 & 18.0 & 5.0 & 1.65 & 1.26 & 1.50 & 15 \\
\hline 32 & 4 & 23.6 & 22.0 & 12.0 & 5.0 & 4.06 & 1.35 & 1.50 & 20 \\
\hline 33 & 4 & 23.6 & 22.0 & 15.0 & 5.0 & 2.86 & 1.30 & 1.50 & 20 \\
\hline 34 & 1 & 23.6 & 26.5 & 23.0 & 5.0 & 1.48 & 1.15 & 1.51 & 60 \\
\hline
\end{tabular}

TABLE 1. List of parameters. $T_{e}, T_{h}$ and $T_{c}$ are the temperatures of the environment (and the initial temperature of the water), heating source and cooling source. Salinity is in units of mol $1^{-1} ; g^{\prime}$ is the reduced acceleration due to gravity $\left(\mathrm{cm} \mathrm{s}^{-2}\right) ; v, \kappa$ are the molecular kinematic viscosity and thermal diffusivity $\left(\mathrm{cm}^{2} \mathrm{~s}^{-1}\right)$.

covered the following range: Prandtl number: 60 to 6600 ; Rayleigh number: $1.5 \times 10^{5}$ to $4.4 \times 10^{7}$.

Miller (1968) made the following heat leakage estimate: for a $5 \mathrm{~K}$ difference between the inner and outer box, the theoretical heat flow by radiation is $5.0 \times 10^{-5} \mathrm{cal} \mathrm{s}^{-1} \mathrm{~cm}^{-2} \mathrm{~K}^{-1}$. The Plexiglas absorption curve peaks sharply in the ultraviolet radiation emitted from the outer surface of the inner box which is absorbed in the inner wall of the outer box; consequently, the heat flow by radiation is further reduced. The measured effective conductivity (conduction + radiation + convection) for Miller's experiments was less than $2.09 \times 10^{-6} \mathrm{cal} \mathrm{s}^{-1} \mathrm{~cm}^{-2} \mathrm{~K}^{-1}$, which had no effect on flow properties.

In order to verify the potential impact of heat leakage through the tank and radiation we carried out two experiments (runs 20 and 21), in which the heating 


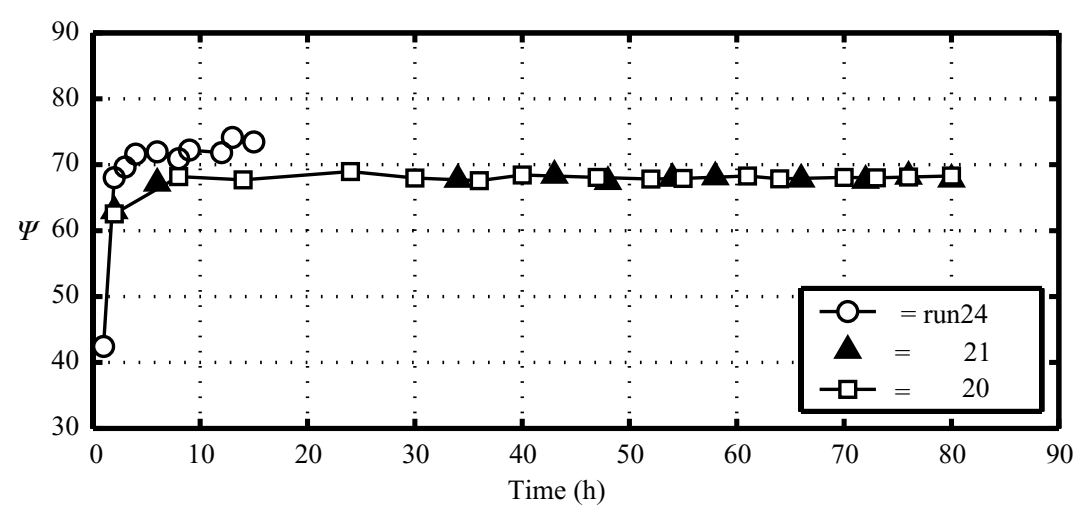

FiguRE 2. Time evolution of the non-dimensional streamfunction maximum, $\Psi=(1 / \kappa) \max \left|\int_{0}^{\delta} \bar{u}(z) \mathrm{d} z\right|$, for 3 runs.

source's temperature was $2 \mathrm{~K}$ higher or lower than the environmental temperature. Circulation rates in these two runs seem nearly identical (figure 2), with the relative error less than $0.5 \%$; thus, we conclude that heat leakage has a minimal impact on the circulation.

The velocity field of the fluid in the tank is measured with a commercial DPIV (digital particle image velocimetry) system of TSI, which contains a double pulsed Nd: YAG laser with a maximum energy output at $120 \mathrm{~mJ}$ per pulse; a synchronizer; a $1.8 \mathrm{~m}$ long laser arm and a digital CCD camera with a spatial resolution of $1280 \times 1024$ pixels and 12-bit intensity resolution. For the technical details of the DPIV technique see Xia, Sun \& Zhou (2003) which describes its application to turbulent thermal convection.

The selection of tracers in fluid is very important in these experiments. In earlier stages of our experiments, hollow glass spheres were used as tracers for experiments with pure water or very low salinity water. However, as many experiments were $20 \mathrm{~h}$ or longer, hollow glass spheres were unsuitable as tracers. In fact, through testing, we found that the copper-salt complex formed in salt water could be used as a suitable tracer. After the copper-salt complex was well-mixed with the fluid, distribution of these tracers was uniform and the particle size shown in the image was smaller than that of the $8 \mu \mathrm{m}$ hollow glass spheres when illuminated by the laser sheet. With INSIGHT 3 software (of TSI), each pair of images taken by the CCD camera was translated into a velocity vector field with a spatial resolution of $1.3 \mathrm{~mm}$ in both horizontal and vertical directions.

In most experiments, the velocity field was measured every hour. Each velocity measurement consisted of 70 images captured within a period of $1 \mathrm{~min}$, from which 69 instantaneous velocity fields were obtained, with an accuracy of two to three digits for the horizontal velocity for most cases; therefore, the mean velocity field, and the deformation of the velocity can be calculated easily. From velocity measurements, we determined two vertical scales. The cell height is defined as the zonal average of distance from the boundary of thermal forcing to the outer edge of the returning flow where the horizontal velocity is $1 \%$ of the maximum horizontal velocity of the returning flow. The boundary-layer thickness is defined as the zonal average of distance from the boundary of thermal forcing to the height where the horizontal velocity declines to $1 \%$ of the maximum horizontal velocity within the boundary layer. 
No water temperature measurements were made. This is due to consideration of thermal isolation. Our tests indicate that even a very thin metal wire connected to a small thermal sensor with the outside instrument can induce noticeable perturbation to the flow field. Thus, investigation of the temperature field should be carried out with non-interfering instruments; however, this is clearly beyond the scope of this study.

Four kinds of salt water (with salt concentrations of $0.25 \mathrm{moll}^{-1}, 0.5 \mathrm{moll}^{-1}$, $3 \mathrm{moll}^{-1}$ and $5 \mathrm{moll}^{-1}$ ) were used in the experiments. Effects of different depths of fluid were also tested in some experiments. The dependence of flow properties on a horizontal length scale was investigated by separating the fluid in the tank with a plexiglas bar inserted into the tank.

A major factor affecting the flow pattern is the vertical position of thermal sources. Four different settings of thermal sources were used in this study: case 1, thermal sources were placed horizontally on the bottom of the fluid; case 2, thermal sources were placed horizontally on top of the fluid; case 3, thermal sources were placed on the bottom and the tank was rotated approximately $12^{\circ}$, so the heating end is $4 \mathrm{~cm}$ below the cooling end; and case 4, similar to case 3 , but the heating end was $4 \mathrm{~cm}$ above the cooling end. For cases 3 and 4, the CCD camera and the coordinates used in analysis were rotated at the same angle as the tank.

\section{Results from experiments}

\subsection{The establishment of circulation}

It is well known that after thermal forcing is switched on, the system normally goes through a transient period before settling down to the final quasi-equilibrium state. The duration of this period depends on many factors, such as the geometry of the tank, forcing, dissipation and the initial state of the system. In general, there is an upper limit of the establishment of the equilibrium state, which is primarily controlled by dissipation processes intrinsic to the system. The time scale for the system to reach a quasi-equilibrium state can be found by performing experiments under different thermal forcing and initial conditions. Our experimental results showed that the dissipation rate was very high and was proportional to the kinetic energy of the flow (figure 3). Such a high dissipation rate implies a relatively shorter time for the system to reach a quasi-equilibrium state.

Note that during the establishment of the solution, the circulation may be energetic and its pattern may appear in forms dramatically different from steady-state solutions. For example, in the case of thermal forcing from the bottom, if the temperature of the heating source is higher than the initial temperature of the water in the tank, there will be a fast-growing plume ascending from the heating source. This plume reaches the top of the tank and drives a very energetic circulation in the tank. Circulation that can penetrate to the whole depth of the tank is called full-penetrating circulation hereinafter. However, for the parameter range discussed in this study such a fullpenetrating circulation cannot be maintained in a steady state. Instead, the final quasi-equilibrium state is characterized by a relatively shallow cell near the boundary of thermal forcing. In this study, such circulation will be called partial-penetrating circulation.

Most previous results obtained from either laboratory experiments or numerical models belong to the category of full-penetration. There are a few exceptions, such as the experimental results obtained by Miller (1968), in which both the partialpenetrating circulation and the full-penetrating circulation results were reported. In the following discussion, Miller's results will be classified into two categories: 


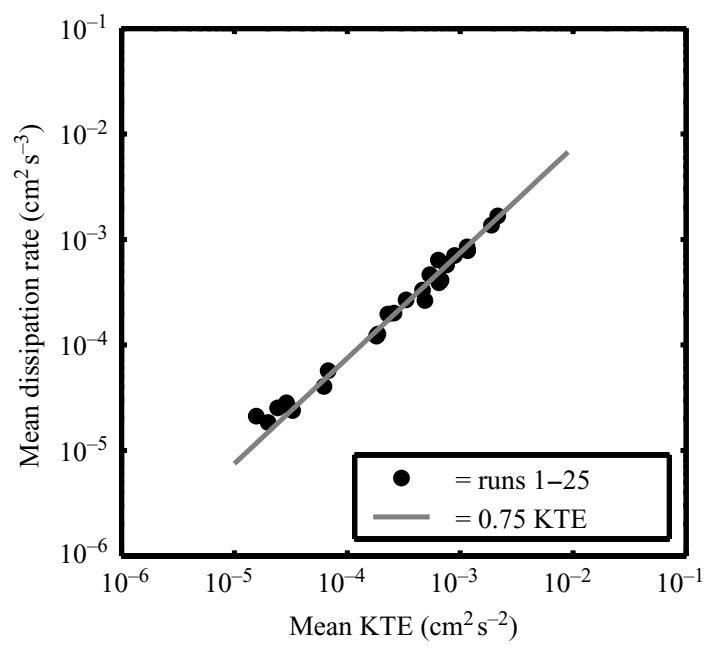

FigurE 3. The dependence of mean dissipation rate on the mean kinetic energy within the cell of the circulation.

full-penetrating solutions which will be labelled as Miller(f) and partial-penetrating solutions which will be labelled as Miller(p). As will be shown shortly, most of our results also belong to the partial-penetrating circulation.

Quasi-equilibrium partial-penetrating circulation can be gradually established if the temperature of the heating source placed on the bottom of the tank is lower than the initial temperature of water in the tank. Beyond expectations, the fluid was set in motion immediately after the thermal sources were turned on. Unlike the case of fullpenetration where the flow was initiated at the heating end of the thermal sources, the initiation of flow for partial-penetrating circulation occurred at the cooling end of the thermal sources owing to the local horizontal gradients in density and pressure. The circulation evolves rapidly within a few hours from the beginning and then evolves gradually to a state close to quasi-equilibrium. As illustrated in figure 2 , the flow system can reach a quasi-equilibrium state within approximately $10 \mathrm{~h}$ for our facilities.

Theoretically, the diffusion time scale can be used to estimate the duration of the establishment of the thermal equilibrium, which is $\tau=H_{0}^{2} / \kappa$, where $H_{0}$ is the water depth and $\kappa$ is the molecular diffusivity. For our setting, $H_{0}=15 \mathrm{~cm}$ and $\kappa=0.0015 \mathrm{~cm}^{2} \mathrm{~s}^{-1}$, so $\tau \simeq 1.7$ days. However, our experiments indicate the system can reach a quasi-equilibrium state within $10-20 \mathrm{~h}$, much shorter than the diffusive time scale because of the high dissipation rate of the system. Special tests run for $80 \mathrm{~h}$ also confirm that the system does not change much after the first 10 to $20 \mathrm{~h}$. Therefore, most of our experiments have been run for at least $10 \mathrm{~h}$ or longer to guarantee the establishment of quasi-equilibrium, as shown in the last column of table 1.

The establishment of quasi-equilibrium circulation for cases 1-3 are similar. There is, however, a minor difference for case 2. Because thermal forcing is now on top of the fluid, the motion starts from the heating source and moves toward the cooling end, thus the time evolution of the circulation is a mirror image of that for case 1 .

The establishment of the circulation for case 4 is slightly different from the other cases. In the first few minutes, a clockwise motion appeared owing to the falling of the cold water along the sloping bottom boundary; this down-slope motion was caused by the temperature of the heating end of the thermal sources being lower than the initial temperature of the fluid. The strength of the clockwise motion decreased 


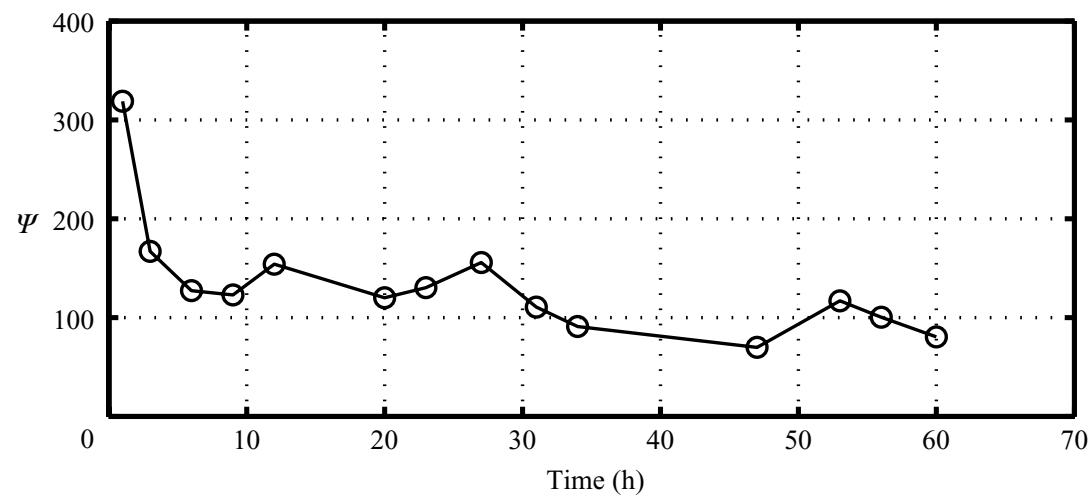

FIGURE 4. The time evolution of non-dimensional streamfunction maximum, $\Psi$, for run 34 .

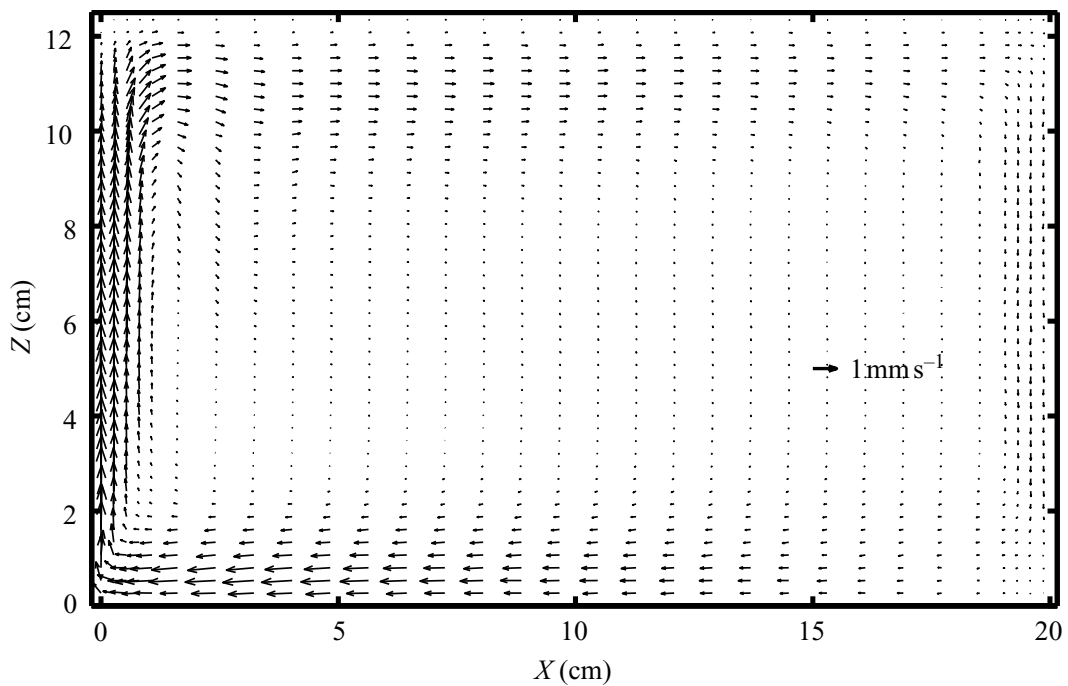

FiguRE 5. Mean velocity field for the case of full-penetrating circulation under horizontal thermal forcing from the bottom of the fluid (run 34), heating at the left, cooling to the right, and time $=27 \mathrm{~h}$.

rapidly as the accumulation of the cold water at the higher pressure level increased, and the anticlockwise circulation cell established gradually over the following few hours.

Although most experiments reached a quasi-equilibrium state, there was an exceptional run which could not reach a quasi-equilibrium state. Run 34 is such an example. This run started with an initial temperature $3 \mathrm{~K}$ colder than the heating end of the thermal source on the bottom. The circulation developed full penetration immediately after starting up and the system went through a continuous transition between full-penetration and partial-penetration. Unlike the circulation of partial penetration, the flow pattern of this run was unstable. Even after $60 \mathrm{~h}$ the circulation was still setting down and oscillating, as indicated by the time evolution of the non-dimensional streamfunction maximum in figure 4.

A snapshot taken at hour 27 is shown in figure 5. Along the left-hand side of the box, a strong thermal jet reached the top of the free surface of the fluid, and a strong 

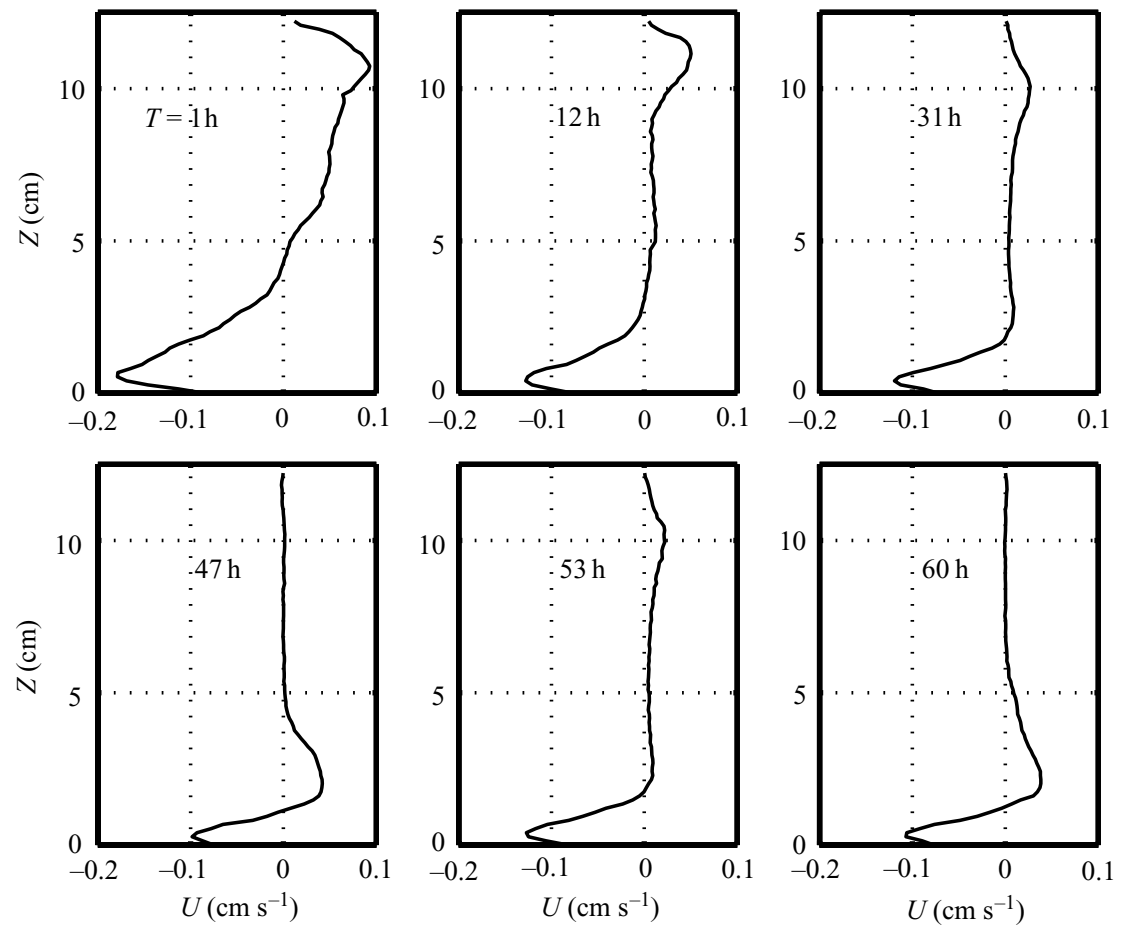

FiguRE 6. Velocity profiles at a section $2.7 \mathrm{~cm}$ from the left-hand sidewall (run 34).

horizontal boundary-layer flow appeared at the bottom and top of the fluid. In the first few hours, the remainder of the fluid gently moved to the bottom, but a distinct velocity boundary layer at the right-hand side of the box was established. During the $60 \mathrm{~h}$ of experiments, 4 types of horizontal return flow were observed, as shown in figure 6; these patterns were repeated twice in this time frame. Since we did not record the velocity field hour by hour, other types of velocity profile may also exist, and the accurate nature of the oscillation remains unclear.

\subsection{Circulation in the quasi-equilibrium states}

The typical quasi-equilibrium mean velocity field for cases 1 to 4 is shown in figure 7. The temperature difference of the thermal sources of these cases is the same, $\Delta T=18.5 \mathrm{~K}$. It is observed that the flow pattern produced by heating/cooling from the top is a mirror image of heating/cooling from the bottom. The pattern in figures $7(c)$ and $7(d)$ is quite different from figures $7(a)$ and $7(b)$. In general, circulation for case 3 is much stronger than that for case 1 ; the circulation cell is rather tall near the heating end, but it is shallow and intense near the cooling end. Case 4 (figure $7 d$ ) is quite different from other cases. The circulation cell for this case is confined to the right-hand half of the tank and no longer occupies the whole length of the tank. In addition, the circulation is separated from the bottom boundary, except near the heating end. Most importantly, the strength of the circulation is greatly decreased.

Since the temperature of the heating end of the thermal sources for most runs of case 1 (runs 1-9,11-21) is lower than the initial temperature of the fluid, the circulation cannot penetrate the entire depth when the depth of the fluid is much larger than the cell height of the corresponding quasi-equilibrium circulation which is approximately $3 \mathrm{~cm}$ in this study. When the temperature of the heating end of the thermal sources is 
(a)

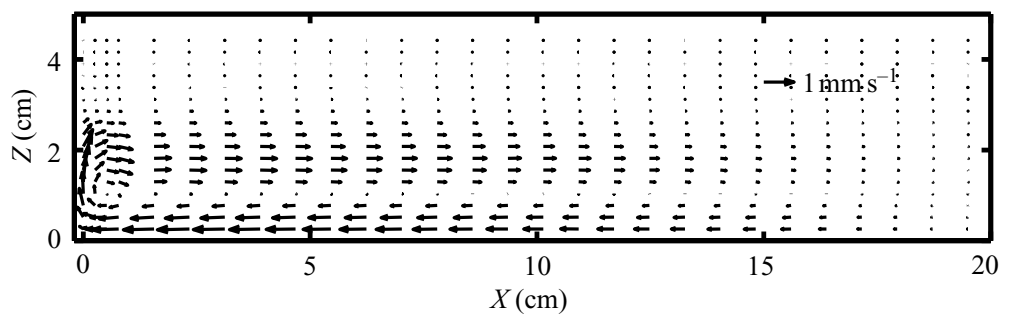

(b)
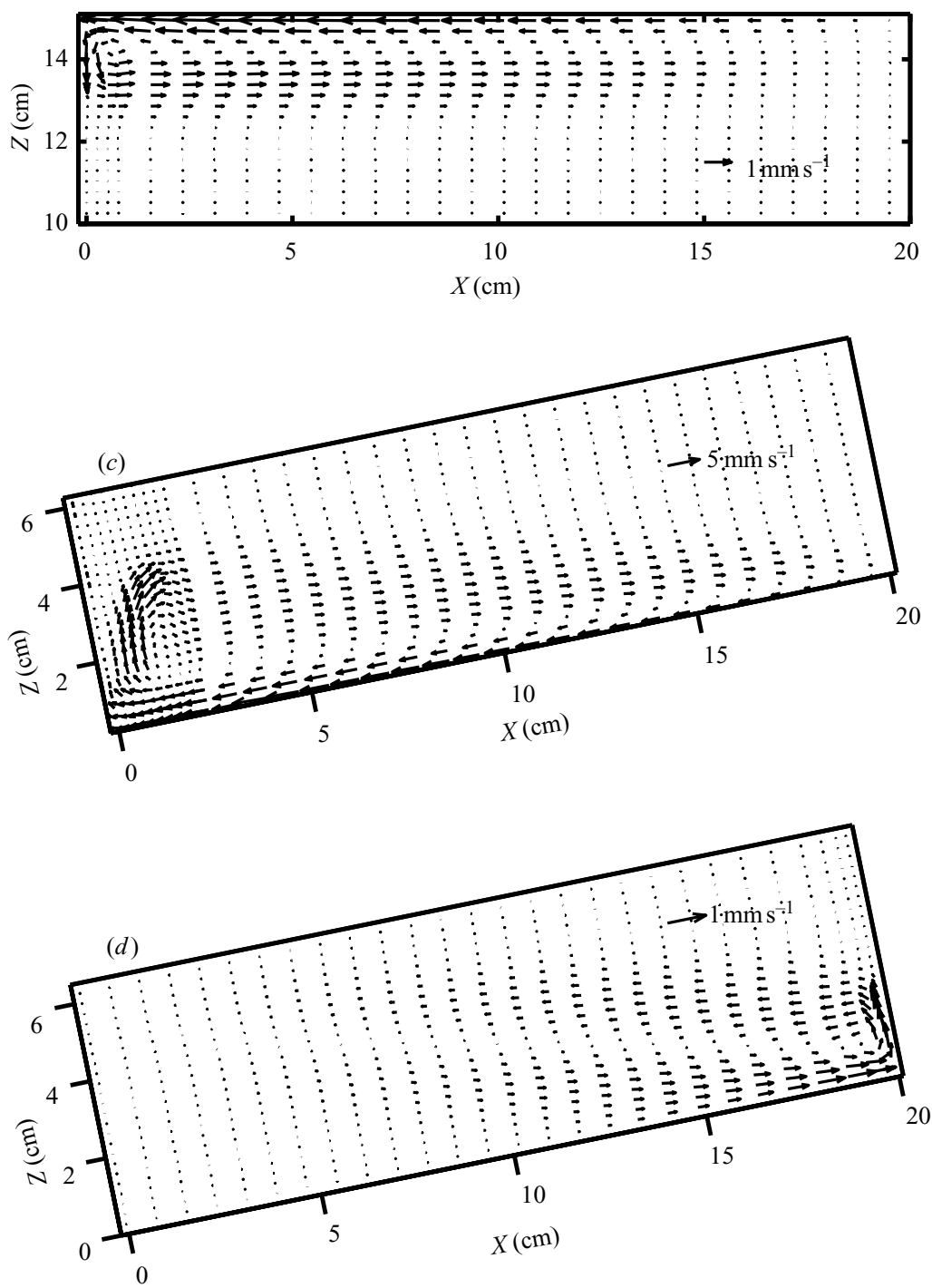

FIGURE 7. Velocity field generated by thermal forcing: $(a)$ case 1 (run 2), heating at the left and cooling at right; $(b)$ case 2 (run 24), heating at the right and cooling at the left; $(c)$ case 3 (run 26), heating at left and cooling at right; $(d)$ case 4 (run 30), heating at right and cooling at left. Bottom is sloping at an angle of $12^{\circ}$ in cases 3 and 4. 
slightly higher than the initial temperature of the fluid, the circulation can penetrate deeper; the strength of the circulation increases accordingly, but this phenomenon can be maintained for only a few hours. In run 10 , the temperature of the heating end of the thermal sources is $1 \mathrm{~K}$ higher than the initial temperature of the fluid. The circulation cell height increases continuously to approximately $7 \mathrm{~cm}$ in the first $7 \mathrm{~h}$ and slowly decreases to an equilibrium state of about $4 \mathrm{~cm}$ after $42 \mathrm{~h}$.

Our experiments show that when the temperature difference between the thermal sources and the initial state of the fluid is large, the circulation reaches the quasiequilibrium state fast. Our laboratory experiments show that this kind of partial penetration is stable. The aspect ratio does not seem to affect cell height or boundarylayer thickness. For example, water depth is only $6.5 \mathrm{~cm}$ for run 1, but the cell height for this run is similar to run 2 in which the depth of the water is doubled, all other parameters have the same value. We also performed a test in which a $1 \mathrm{~cm}$ thick plate was inserted into the tank, so that the width of the tank was reduced to $1.5 \mathrm{~cm}$. Under the same forcing condition, the circulation pattern seems to be insensitive to change in the width of the tank.

\subsection{Characters of the flow}

In most instances, flow observed in these experiments is laminar with complicated time-dependent structures. Although the flow may look turbulent, it does not satisfy the strict definition of turbulence, as discussed by Paparella \& Young (2002). For the circulation shown in figure $7(a)$, the corresponding velocity deformation $\left\langle\|\nabla \overline{\boldsymbol{u}}\|^{2}\right\rangle$ and root mean square (r.m.s.) perturbation velocity $u_{r m s}=\sqrt{\overline{u^{\prime 2}+w^{\prime 2}}}$ are shown in figures $8(a)$ and $8(b)$. Note that at the lower left-hand corner there is a strong thermal plume and both dissipation and the velocity fluctuations are strongest. Additionally, a secondary dissipation maximum exists in the interior, located at the level of the maximal vertical shear (figure $7(a)$ ). Also, the relative strength of the perturbation velocity, $\sqrt{u^{\prime 2}+w^{\prime 2}} / \sqrt{\bar{u}^{2}+\bar{w}^{2}}$, is shown in figure $8(c)$. It can be readily seen that the relative strength is maximal along the outer edge of the circulation cell where the temporal mean velocity is slow and the velocity fluctuations are relatively strong. The narrow zone of local maximum of this ratio at the middle level of the circulation cell is due to the weak mean flow there.

As discussed in the Appendix, the total dissipation rate consists of two terms $\varepsilon_{\text {total }}=\varepsilon_{m}+\varepsilon_{t}$ : the first term is due to the mean velocity deformation, $\varepsilon_{m}=v\left\langle\|\nabla \overline{\boldsymbol{u}}\|^{2}\right\rangle_{\text {cell }}$, and the second term is due to the fluctuated velocity, $\varepsilon_{t}=15 v\left\langle\left(\partial u^{\prime} / \partial x\right)^{2}\right\rangle_{\text {cell }}$. Both the total dissipation rate and the r.m.s. perturbation velocity of the flow measured in the experiments span a large range (figure 9). The dissipation rate increases two decades, indicating that the strength of the fluctuated velocity intensifies as the Rayleigh number increases. Apparently, there is a transition taking place near a critical Rayleigh number $R a_{L} \simeq 5 \times 10^{8}$. For Rayleigh number below this transition zone, both the total dissipation rate and the r.m.s. perturbation velocity are relatively small; however, above this transition zone, there is a rapid increase in both these properties.

Another set of flow properties is the bulk mixing coefficient within the circulation cell defined as $\kappa_{t}=U h_{c}^{2} / L$ (assuming vertical diffusion is balanced by vertical advection, where $L$ is the length of the tank, $h_{c}$ is the cell height of the circulation and $U$ is the horizontal velocity). Such a transition can also be inferred from the dependence of the bulk diffusivity on the Rayleigh number (figure 10). It can be readily seen that for low Rayleigh number, the ratio of the bulk mixing coefficient vs. molecular mixing coefficient is roughly a constant between 1 to 2 ; however, for 
(a)
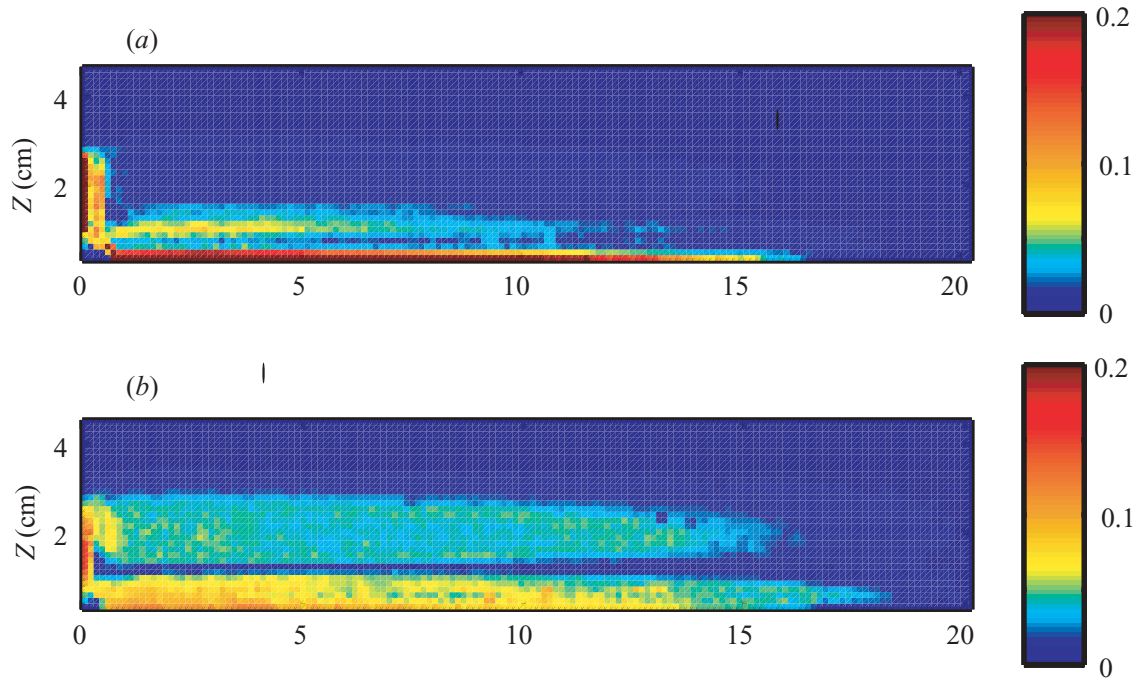

(c)
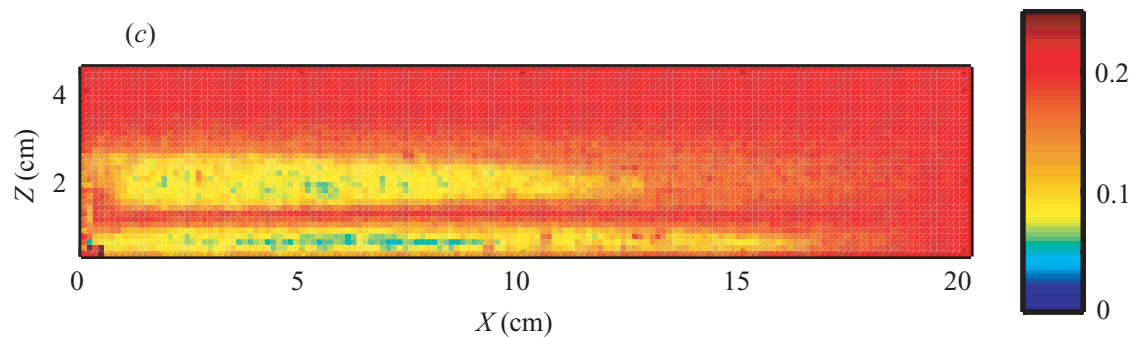

Figure 8. Flow properties for run 2. (a) The velocity deformation, $\left\langle\|\nabla \overline{\boldsymbol{u}}\|^{2}\right\rangle_{\text {cell }}$, in $1 / s^{2} ;(b)$ the r.m.s perturbation velocity in $\mathrm{cm} \mathrm{s}^{-1} ;(c)$ the ratio of r.m.s velocity and local mean velocity.
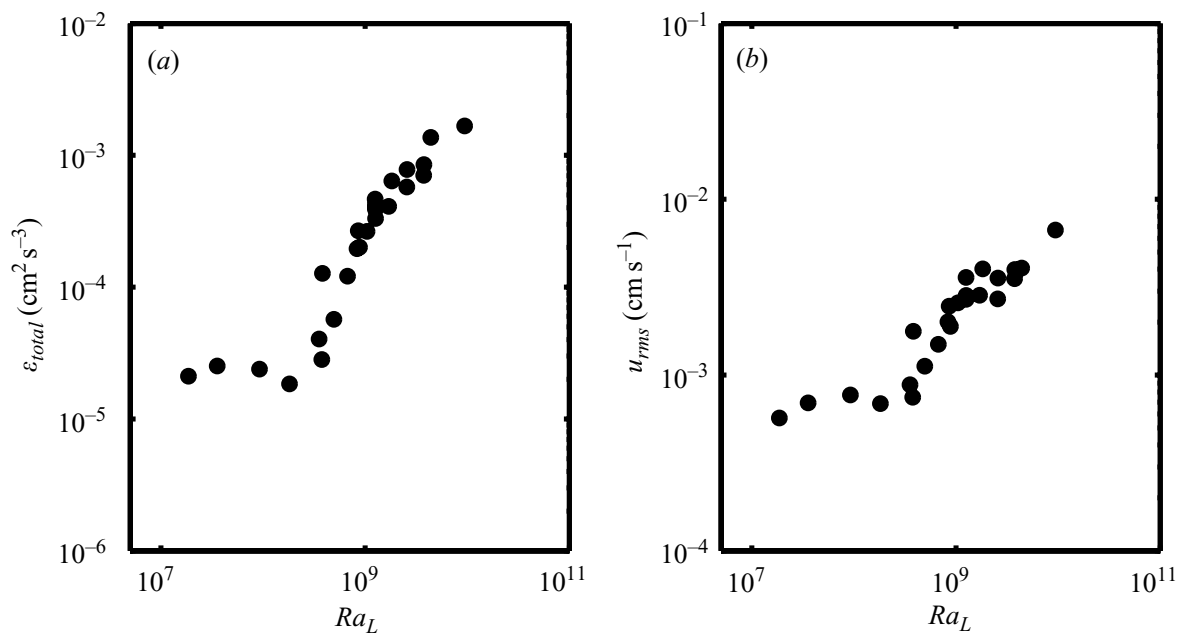

FIGURE 9. Total energy dissipation rate and r.m.s perturbation velocity $u_{r m s}=\sqrt{\overline{u^{\prime 2}+w^{\prime 2}}}$, as functions of Rayleigh number for runs 1-25. 


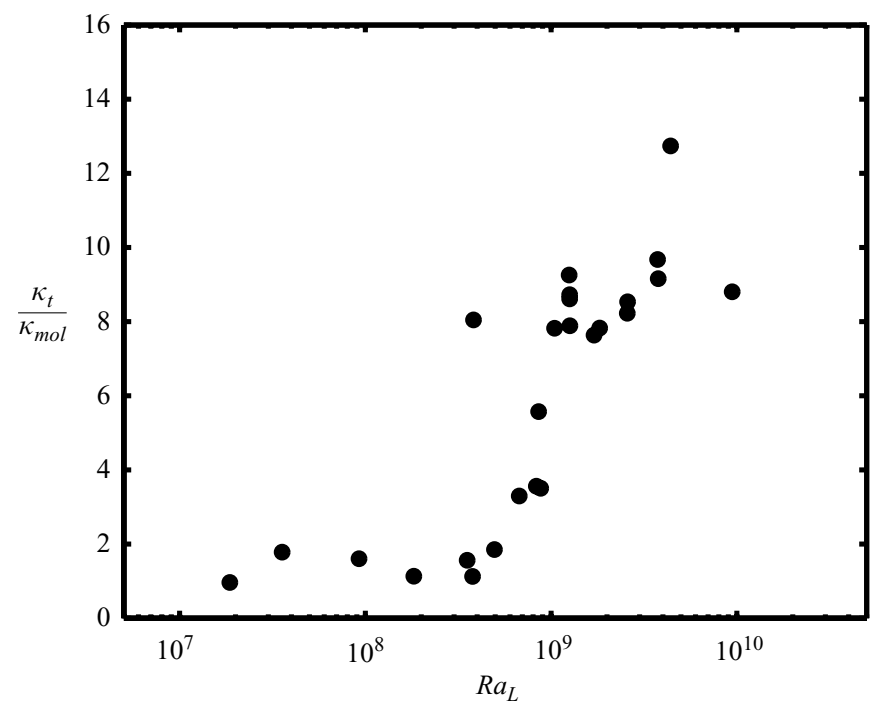

FIGURE 10. Ratio of bulk diffusivity vs. molecular diffusivity for runs 1-25.

Rayleigh number larger than the critical value, $R a_{L} \simeq 5 \times 10^{8}$, this ratio increases rapidly, and eventually reaches the value of 10 to 14 (figure 10). Such an abrupt change in the ratio of mixing coefficient implies that the large velocity fluctuations can also cause considerable mixing even when the flow is in the laminar range.

\subsection{Scaling laws of circulation}

Thermal-driven circulation can be characterized in terms of the horizontal Rayleigh number, $R a_{L}=g^{\prime} L^{3} / v \kappa$, where $g^{\prime}=g \alpha \Delta T$ ( $\Delta T$ is the temperature difference of the cooling and heating sources), $\kappa$ and $v$ are molecular diffusivity and viscosity of the fluid, respectively. Alternatively, we can also use the vertical Rayleigh number $R a_{\delta}=g^{\prime} \delta^{3} / \nu \kappa$, where $\delta$ is the thickness of the velocity boundary layer adjacent to the heating/cooling surface. Both the molecular diffusivity $\kappa$ and viscosity $v$ are calculated according to a formula discussed by Siedler \& Peters (1986). Note that the salinity of water used in these experiments is beyond the original range where these formulae were defined. Our experiments were carried out under room temperature and both $\kappa$ and $v$ seem insensitive to changes in salinity. Since there are no other formulae available, these formulae were used in this study. For all our experiments, the other important non-dimensional number, the Prandtl number, is $\sigma=v / \kappa \approx 8$ and varies only slightly.

The most important quantity describing the circulation is the non-dimensional streamfunction maximum, $\Psi=\tilde{\Psi} / \kappa$, where $\tilde{\Psi}$ is the maximum of the dimensional streamfunction defined as $\tilde{\Psi}=\max \left|\int_{0}^{\delta} \bar{u}(z) \mathrm{d} z\right|$. Results from our experiments for the horizontal thermal forcing cases (runs 1-25) are consistent with the classical 1/5power law first discussed by Rossby (1965) (figure 11). As discussed above, flow transition takes place within the parameter regime of our experiments; thus, to fit the observations, the scaling power law appears in the form of two segments. For example, the dependence of the non-dimensional streamfunction, $\Psi$, on $R a_{L}$ for case 1 showed a distinct offset in constant (the difference of the constant for the two segments is as large as 5 times), but remains the same 1/5-power (figure 11a), since the flow holds laminar in these two segments. 

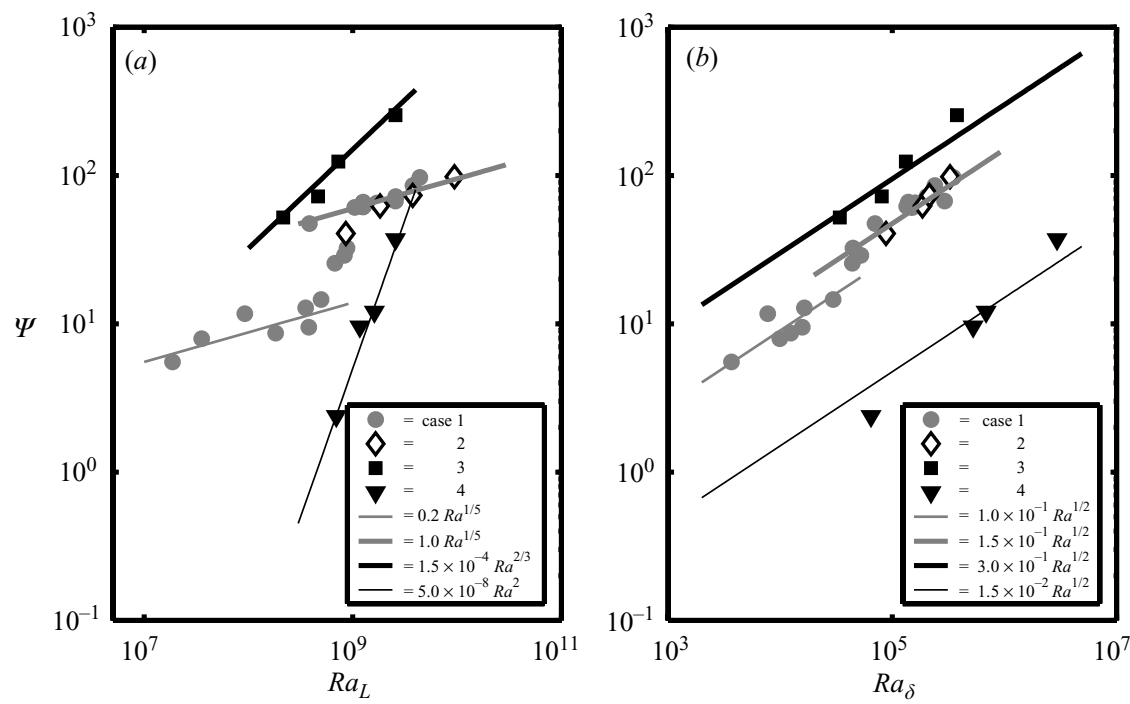

FiguRE 11. The relationship between non-dimensional streamfunction maximum, $\Psi$, and Rayleigh number, for runs 1-33. (a) horizontal Rayleigh number; $(b)$ vertical Rayleigh number.

It is also readily seen that the circulation in cases 1 and 2 obeys the same 1/5power law; thus, within the parameter range discussed in this study, horizontal heating/cooling on the top or bottom of the tank produces virtually the same circulation pattern.

On the other hand, the least-squares fitting for cases 3 and 4 gives rise to different power laws (figure 11a). Although no flow transition appears for the results obtained from cases 2, 3 and 4, it is speculated that such transition may take place, if we run these experiments over a wider range of parameters.

The dependence of the overturning rate on $R a_{\delta}$ is quite different from that on $R a_{L}$ (figure $11 b$ ), the overturning rates from experimental results for all cases seem to obey the same power law, but with different constants. Although the transition for case 1 still exists, it is no longer obvious, the difference of the constant is reduced to 1.5 times for the two segments. The 1/2-power law in the $\Psi-R a_{\delta}$ relationship which is consistent with the $1 / 5$-power law in the $\Psi-R a_{L}$ relationship can also fit all experimental data well. The difficulty in using $R a_{\delta}$ instead of $R a_{L}$ is that the boundarylayer thickness cannot be given a priori.

We can see from table 2 that both the boundary-layer thickness, $\delta$, and the circulation cell height, $h_{c}$, vary slightly within the parameter regime of our experiments. As shown in figure 12( $a)$, the dependence of the boundary-layer thickness is consistent with the $-1 / 5$-power law of Rossby (1965) in two segments. The dependence of the circulation cell height on $R a_{L}$ is similar to that of the boundary-layer thickness and fits a $-1 / 5$-power law (figure 12b). There is no perfect fit, and data scattering exists that may be due to experimental errors (for the spatial resolutions of $1.3 \mathrm{~mm}$ in this study, the relative errors for $\delta$ and $h_{c}$ are $11 \%-25 \%$ and $3 \%-8 \%$, respectively). Note that in previous studies, such as Rossby $(1965,1998)$, the circulation occupied the whole depth of the tank; thus, cell height does not vary in these cases. In fact, he did not discuss the dependence of cell height on circulation parameters. 


\begin{tabular}{|c|c|c|c|c|c|c|c|c|c|}
\hline $\begin{array}{l}\text { Run } \\
\text { number }\end{array}$ & $L$ & $H_{0}$ & $h_{c}$ & $\delta$ & $\Psi$ & $U \times 10^{2}$ & $\varepsilon_{m} \times 10^{5}$ & $\varepsilon_{t} \times 10^{5}$ & $\left\langle\sqrt{\overline{u^{\prime 2}+w^{\prime 2}}}\right\rangle \times 10^{3}$ \\
\hline 1 & 20.0 & 6.5 & 3.18 & 0.96 & 66.22 & 2.44 & 19.96 & 19.05 & 3.75 \\
\hline 2 & 20.0 & 13.0 & 3.29 & 1.00 & 65.03 & 2.25 & 19.67 & 22.17 & 4.01 \\
\hline 3 & 20.0 & 13.0 & 3.13 & 0.97 & 67.24 & 2.48 & 35.78 & 21.65 & 3.86 \\
\hline 4 & 20.0 & 13.0 & 3.51 & 0.86 & 97.03 & 3.10 & 95.98 & 40.65 & 5.91 \\
\hline 5 & 20.0 & 13.0 & 3.27 & 0.86 & 71.91 & 2.40 & 47.74 & 30.43 & 5.28 \\
\hline 6 & 20.0 & 13.0 & 2.91 & 0.80 & 85.46 & 3.24 & 53.83 & 31.30 & 5.96 \\
\hline 7 & 20.0 & 13.0 & 2.27 & 0.72 & 12.79 & 0.91 & 2.17 & 1.87 & 1.19 \\
\hline 8 & 20.0 & 13.0 & 2.45 & 0.74 & 32.34 & 1.76 & 11.21 & 8.87 & 2.69 \\
\hline 9 & 20.0 & 13.0 & 3.41 & 0.95 & 61.83 & 2.03 & 12.59 & 20.59 & 4.21 \\
\hline 10 & 20.0 & 13.0 & 4.64 & 1.14 & 47.44 & 1.12 & 4.10 & 8.61 & 2.90 \\
\hline 11 & 8.8 & 13.0 & 1.71 & 0.51 & 5.52 & 0.43 & 1.10 & 1.01 & 0.92 \\
\hline 12 & 10.2 & 13.0 & 2.27 & 0.67 & 7.94 & 0.53 & 1.27 & 1.25 & 1.07 \\
\hline 13 & 20.0 & 13.0 & 2.22 & 0.69 & 9.45 & 0.68 & 1.31 & 1.52 & 1.11 \\
\hline 14 & 20.0 & 13.0 & 2.37 & 0.78 & 14.58 & 0.99 & 2.66 & 3.05 & 1.64 \\
\hline 15 & 20.0 & 13.0 & 2.51 & 0.81 & 25.58 & 1.56 & 7.22 & 4.88 & 1.95 \\
\hline 16 & 20.0 & 13.0 & 2.51 & 0.80 & 28.95 & 1.69 & 9.37 & 10.23 & 2.95 \\
\hline 17 & 20.0 & 13.0 & 2.49 & 0.82 & 8.65 & 0.54 & 0.66 & 1.18 & 1.09 \\
\hline 18 & 20.0 & 13.0 & 2.68 & 0.88 & 11.67 & 0.67 & 0.94 & 1.45 & 1.11 \\
\hline 19 & 20.0 & 12.0 & 2.98 & 0.91 & 65.13 & 2.48 & 23.46 & 17.52 & 4.04 \\
\hline 20 & 20.0 & 13.0 & 3.26 & 0.99 & 61.29 & 2.12 & 13.61 & 31.27 & 5.59 \\
\hline 21 & 20.0 & 13.0 & 3.24 & 0.98 & 60.90 & 1.98 & 12.86 & 13.57 & 3.69 \\
\hline 22 & 20.0 & 15.0 & 3.06 & 0.94 & 40.55 & 1.78 & 10.92 & 15.84 & 3.85 \\
\hline 23 & 20.0 & 15.0 & 3.19 & 0.93 & 62.34 & 2.32 & 20.61 & 43.34 & 7.45 \\
\hline 24 & 20.0 & 15.0 & 3.17 & 0.77 & 73.35 & 2.91 & 37.20 & 33.26 & 5.54 \\
\hline 25 & 20.0 & 15.0 & 2.46 & 0.65 & 98.09 & 4.44 & 99.47 & 67.90 & 12.40 \\
\hline 26 & 20.0 & 13.0 & 4.08 & 1.06 & 254.74 & 7.68 & 525.43 & 557.98 & 25.23 \\
\hline 27 & 20.0 & 12.0 & 3.71 & 1.12 & 72.31 & 2.77 & 18.04 & 8.38 & 5.18 \\
\hline 28 & 20.0 & 12.0 & 3.88 & 1.13 & 124.03 & 4.25 & 66.74 & 19.79 & 7.67 \\
\hline 29 & 20.0 & 12.0 & 3.81 & 1.08 & 52.04 & 2.03 & 13.57 & 6.74 & 4.50 \\
\hline 30 & 20.0 & 13.0 & 5.92 & 2.09 & 37.05 & 0.93 & 2.86 & 5.17 & 2.99 \\
\hline 31 & 20.0 & 12.0 & 3.22 & 0.91 & 2.38 & 0.14 & 0.05 & 0.10 & 0.44 \\
\hline 32 & 20.0 & 12.0 & 4.41 & 1.51 & 12.01 & 0.41 & 0.50 & 0.92 & 1.23 \\
\hline 33 & 20.0 & 12.0 & 3.76 & 1.53 & 9.55 & 0.31 & 0.22 & 0.45 & 0.95 \\
\hline 34 & 20.0 & 13.0 & & & & Unstea & solution & & \\
\hline
\end{tabular}

TABLE 2. Results of experiments. $L, H_{0}, h_{c}$ and $\delta$ are horizontal lengths of the tank, depth of the water, and height of the thermal circulation cell, and boundary-layer thickness, respectively; $\Psi=(1 / \kappa) \max \left|\int_{0}^{\delta} \bar{u}(z) \mathrm{d} z\right|$ is the maximal non-dimensional streamfunction, $\bar{u}$ is the arithmetical mean of the positive horizontal velocity (ensemble mean) in the circulation cell $\left(\mathrm{cm} \mathrm{s}^{-1}\right) . \varepsilon_{m}=v\left\langle\|\nabla \overline{\boldsymbol{u}}\|^{2}\right\rangle_{\text {cell }}$ is the ensemble mean of the dissipation rate averaged over the volume of the circulation cell for temporal mean velocity and $\varepsilon_{t}=15 v\left\langle\left(\partial u u^{\prime} / \partial x\right)^{2}\right\rangle_{\text {cell }}$ is the ensemble mean of the dissipation rate averaged over the volume of the circulation cell for fluctuated velocity.

\subsection{Comparison with previous results}

To cover a wide range in the Rayleigh number, Rossby (1965) used a fixed temperature difference of approximately $10 \mathrm{~K}$, but varied the kinematic viscosity over three orders of magnitude. Thus, the Rayleigh number and Prandtl number varied over three orders of magnitude. Miller (1968) used three temperature differences (4.5, 6.5, and $8 \mathrm{~K}$ ) in the thermal sources and varied the kinematic viscosity and the Prandtl number over two orders of magnitude. Mullarney et al. (2004) used pure water in their experiments, in which the Prandtl number varied from 3.95 to 10 . The Rayleigh number varied 

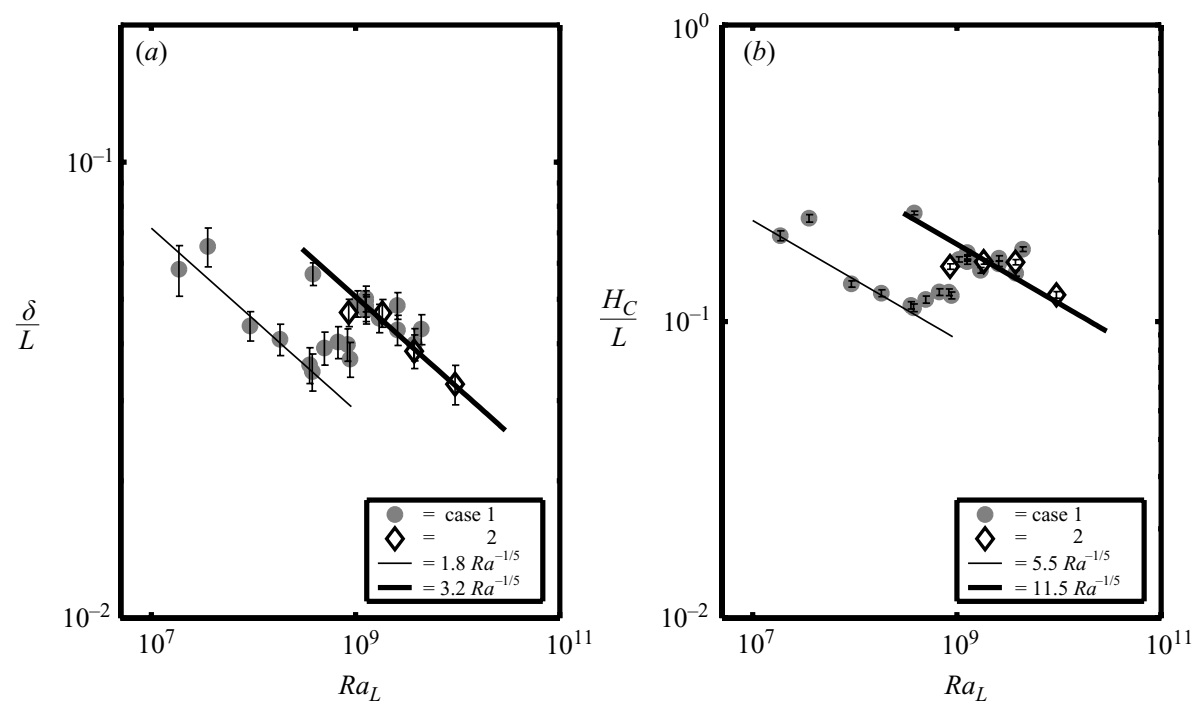

FIGURE 12. The dependence of non-dimensional vertical height on the Rayleigh number for runs 1-25. (a) Non-dimensional boundary-layer thickness; $(b)$ non-dimensional cell height.

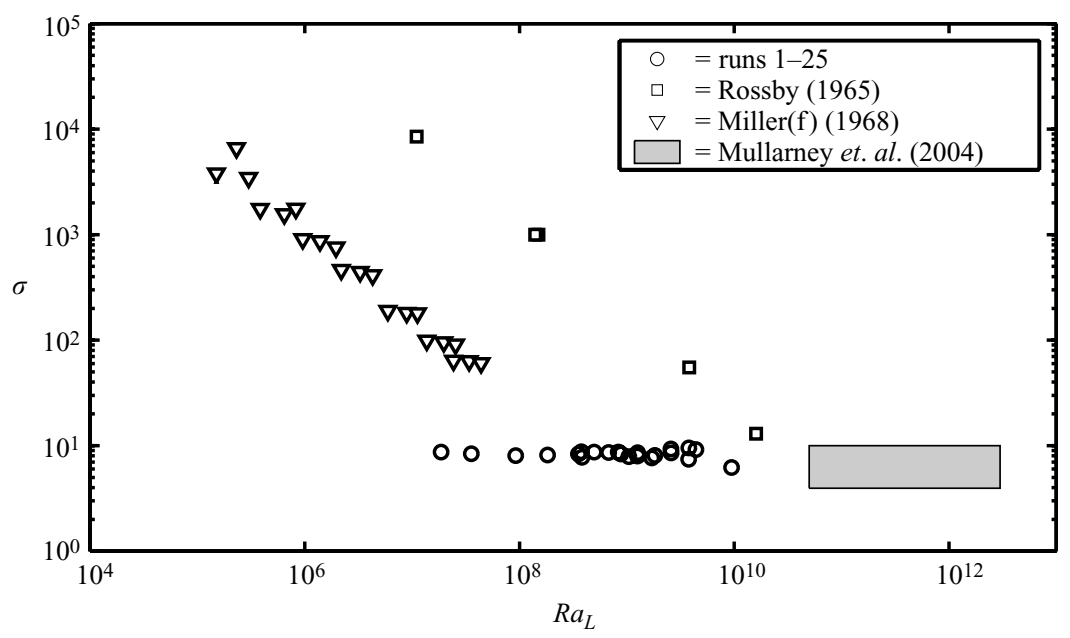

FIGURE 13. Sketch of the range of Rayleigh number and Prandtl number for all the previous experiments and this study.

roughly two orders by changing the heat flux. In our study, the temperature difference of the thermal sources varies from $0.5 \mathrm{~K}$ to $38 \mathrm{~K}$, so the Rayleigh number changes greatly, but the Prandtl number remains nearly constant. A sketch of the range of Rayleigh number and Prandtl number for all these experiments is shown in figure 13.

For the dependence of boundary-layer thickness on $R a_{L}$, the above mentioned experiments are illustrated in figure 14. The results of Rossby (1965) fit the $-1 / 5$ power law with a constant of 1.3; the results of Miller (1968) fit a -0.12-power law with a constant of 1.0 and the results of Mullarney et al. (2004) fit the -1/5-power law with a constant of 2.759. Since the boundary-layer thickness for the case of 


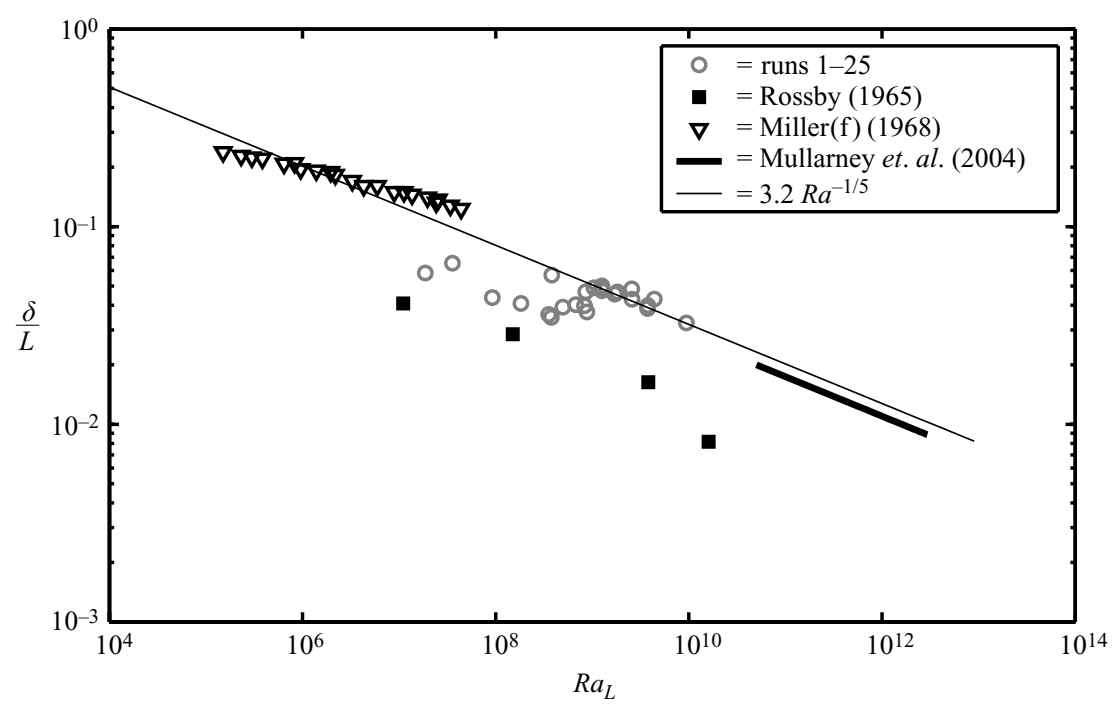

FIGURE 14. Comparison of the dependence of non-dimensional boundary-layer thickness on Rayleigh number for all experimental results related to horizontal convection.

partial-penetration was not given in Miller's tables, only full-penetration results are cited here.

Considering the errors of measurement, our results for the high-Rayleigh-number range $\left(R a_{L}>5 \times 10^{8}\right)$ are consistent with the results of Mullarney et al. (2004) which operated at larger Rayleigh number. The smaller constant of Rossby's (1965) result within the same range of Rayleigh number may be caused by the high viscous fluid. Although the Miller(f) results are quite different from all other experimental results in power law, they follow the same trend and maybe it is the properties of the flow for low Rayleigh number.

For all the experiments mentioned above, the dependence of the overturning rate on $R a_{L}$ is shown in figure $15(a)$ which is greatly scattered. The results of Rossby (1965) fit the 1/5-power law with a constant of 0.4; the results of Miller(f) as a whole fit a 1/3-power law with a constant of 0.3 and the results of Mullarney et al. (2004) fit the $1 / 5$-power law with a constant of 1.158 .

Our results for Rayleigh number larger than the transition are consistent with those of Mullarney et al. (2004). Rossby's (1965) results are located between the two segments of this study and Miller's (1968) results seem to have no relation to other datasets.

Note that the Prandtl number may play an important role in controlling the circulation if a different fluid is used. If the non-dimensional streamfunction maximum is based on $v$, i.e. the non-dimensional streamfunction maximum is defined as $\Psi / \sigma$, and the Grashof number $G r_{\delta}=g^{\prime} \delta^{3} / \nu^{2}=R a_{\delta} / \sigma$ is used instead of the Rayleigh number, the highly scattered data in figure $15(a)$ will become a tightly clustered trend (figure $15 b$ ). For larger Grashof number, it fits a 1/2-power law which is consistent with the scaling of Rossby (1965). For smaller Grashof number, it fits a higher power law (in figure $15 b$ it seems to fit a 3/4-power law) which is consistent with the numerical results as described by Somerville (1967), Beardsley \& Festa (1972) and Siggers et al. (2004) that there is a smooth transition in the $\Psi-R a$ relationship from proportional 

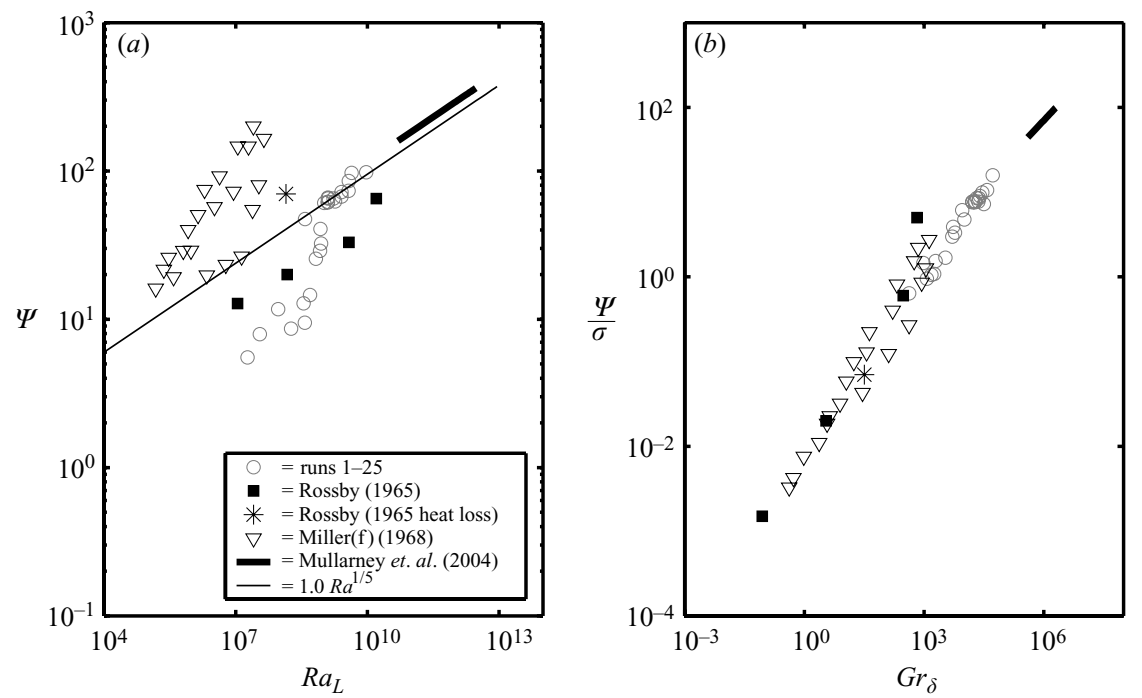

FiguRE 15. (a) The dependence of overturning rate on Rayleigh number for all the experimental results. $(b)$ The relationship between $\Psi / \sigma$ and the Grashof number for this study (runs 1-25) and previous studies. The star indicates the special case with heat loss in Rossby's experiments.

for small Rayleigh number toward a fractional power-law dependence for moderate Rayleigh number.

The star in figure 15 indicates the case with heat loss in Rossby's (1965) experiments. Apparently, the dependence of overturning rate on Grashof number fits data from cases of either partial-penetrating circulation (this study) or full-penetrating circulation (Rossby 1965, Miller 1968; Mullarney et al. 2004); in addition, it is insensitive to the type of thermal forcing and it is even insensitive to the case with heat loss through the side boundaries discussed by Rossby (1965).

\subsection{Dissipation rate}

Paparella \& Young (2002) discussed an anti-turbulence theorem and obtained an equality between the mechanical energy generation rate and the dissipation rate due to mean flow. As described in previous sections, the flow driven by horizontal convection consists of a large degree of fluctuation at large Rayleigh number, and the velocity fluctuations change the flow properties greatly, although the flow remains laminar. Thus, as discussed in the Appendix, we modified the formula for the balance between the mechanical energy generation rate and the dissipation rate of Paparella \& Young (2002), including the effects of velocity fluctuations. The total dissipation rate within the circulation cell composed of two items: $\varepsilon_{m}$ and $\varepsilon_{t}$. Note that there is a close relationship between the total dissipation rate and the dissipation rate according to the mean velocity deformation, $\varepsilon_{\text {total }}=\varepsilon_{m}+\varepsilon_{t} \simeq 2 \varepsilon_{m}$ (figure 16).

Theoretically, the mechanical energy generation rate is equal to the total dissipation rate, i.e. $\varepsilon_{\text {total }} /\left(\langle p \nabla \cdot \boldsymbol{u}\rangle_{\text {cell }} / \rho_{0}\right)=1$. Figure 17 shows the dependence of $\varepsilon_{\text {total }} /\left(\kappa g^{\prime} / 2 h_{c}\right)$ on $R a_{L}$. The value of $\varepsilon_{\text {total }} /\left(\kappa g^{\prime} / 2 h_{c}\right)$ is much smaller than unity for small Rayleigh number and for Rayleigh number larger than the critical value this ratio increased to about 0.5 .

Note that the rate of mechanical energy generation within the cell, $\kappa g^{\prime} / 2 h_{c}$, is obtained under the assumption that $\bar{T}_{\text {top }} \simeq T_{h}$. In fact, $\bar{T}_{\text {top }} \leqslant T_{h}$, thus, $\kappa g^{\prime} / 2 h_{c}$ should 


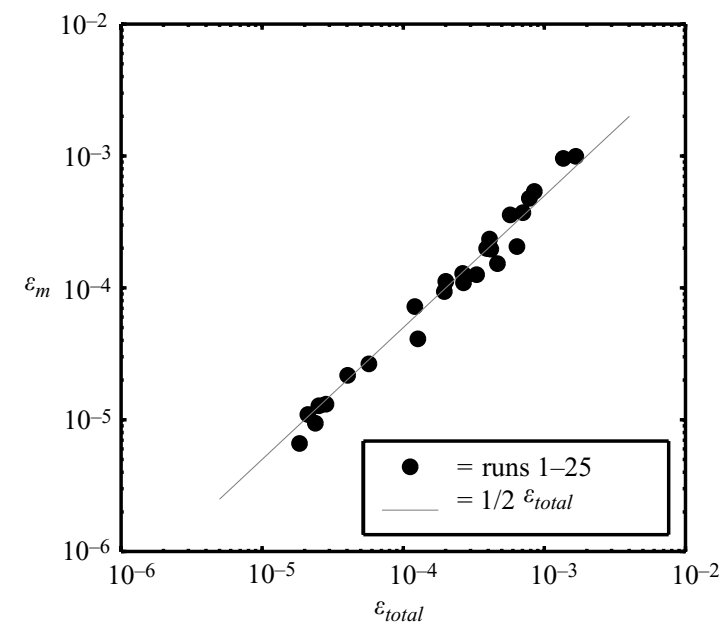

FIGURE 16. The relation between total energy dissipation rate and energy dissipation rate calculated from mean velocity for runs $1-25$.

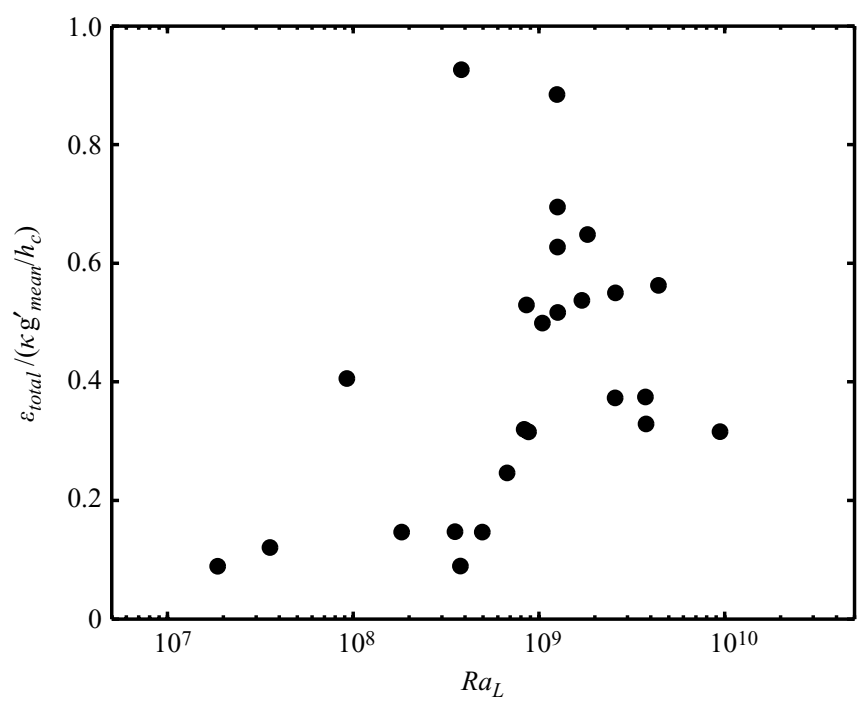

FIGURE 17. Ratio of total energy dissipation rate and buoyancy flux diagnosed from experiments for runs $1-25$.

be interpreted as the upper bound of the dissipation rate. The difference between $\bar{T}_{t o p}$ and $T_{h}$ can induce non-negligible errors for high-speed circulation (or large Rayleigh number). For example, in run $2 T_{h}=22.5^{\circ} \mathrm{C}$ and the ratio of $\varepsilon_{\text {total }} /\left(\kappa g^{\prime} / 2 h_{c}\right)$ is 0.72 , the measured mean temperature at the top boundary is $\bar{T}_{t o p}=20.1^{\circ} \mathrm{C}$ and the ratio of $\varepsilon_{\text {total }} /\left(\kappa \alpha g / h_{c}\right)\left(\bar{T}_{\text {top }}-\bar{T}_{\text {bottom }}\right)$ is 0.97 , the result is much improved. However, for the low-speed circulation (or small Rayleigh number) the assumption of $\bar{T}_{\text {top }}=T_{h}$ is quite accurate. For example, in run $16, T_{h}=21.0^{\circ} \mathrm{C}, \bar{T}_{\text {top }}=20.8^{\circ} \mathrm{C}$ and the ratio between energy dissipation rate and energy generation rate within the cell is 0.32 and 0.35 for the two definitions above, respectively.

Experimental results indicate that for the range of Rayleigh number larger than $5 \times 10^{8}$ (in this study only) the ratio between energy dissipation rate and energy 
generation rate seems consistent with the theory of Paparella \& Young (2002). How ever, for the case of low Rayleigh number, the discrepancy is quite large. There are many potential sources of error. For example, the assumption of hydrostatic pressure may introduce errors, but no estimations are available at present. Thus, the reason for the discrepancy between the theory of Paparella \& Young and our laboratory experiments for small Rayleigh number remain unclear.

\section{Discussion and conclusion}

Our laboratory experiments indicate that horizontal differential heating can always drive a circulation, including cases of heating/cooling from the top or bottom of the tank. Even in the case of a sloping bottom with heating at a pressure level lower than cooling, thermal circulation exists and it can be observed with the naked eye. More importantly, results suggest that for the parameter range in this study, stable thermal circulation appears in the form of a shallow cell (or boundary layer) adjacent to the boundary where thermal forcing is applied. Therefore, consistent with many previous studies based on theoretical reasoning, numerical experiments and laboratory experiments (Jeffreys 1925; Rossby 1965; Paparella \& Young 2002; Mullarney et al. 2004), results from our experiments have further confirmed that the so-called Sandström theorem is inaccurate. In fact, purely thermal forcing can drive circulation in the ocean, although such circulation may be rather slow; nevertheless, the ocean can theoretically work as a heat engine with an extremely low thermal efficiency.

As discussed above, with the increase of the Rayleigh number, the velocity fluctuations intensify, more thermal energy can flow into the fluid, resulting in more mechanical energy production and stronger circulation. Since, in this case, there is no external source of mechanical energy, the system must be self-organized in a way that generates mechanical energy internally, i.e. dissipation, especially that caused by velocity fluctuations, is responsible for setting up the density field in such a way that it guarantees continuous conversion from internal energy to mechanical energy.

There is speculation about what might happen, if a basin the size of the Atlantic is subject to thermal forcing. Assuming the rate of mixing remains on the level of molecular mixing, existing theory predicts weak circulation and a thin main thermocline (e.g. Stern 1975). Using the upper bound of energy generation rate defined by Paparella \& Young (2002), the total conversion rate from internal energy associated with surface thermal forcing to mechanical energy is about $1.5 \mathrm{GW}$, which is too small to support the meridional circulation in the ocean. Note that geothermal heating on the sea floor can generate mechanical energy of the order of $0.05 \mathrm{TW}$, which is much larger than that due to surface thermal forcing. Mechanical energy input to the world oceans from wind and tidal dissipation energy is estimated as $64 \mathrm{TW}$ (Huang 2004). Thus, mechanical energy generated from surface thermal forcing is at least 10000 times smaller than the mechanical energy generated by wind and tidal dissipation. The hypothetical circulation driven by surface thermal forcing alone in the ocean is extremely weak, so it may be totally negligible in comparison with the strong meridional overturning circulation observed in the oceans, which is apparently driven by the stirring of wind and tidal dissipation.

Our experiments indicate that the rate of bulk mixing driven by horizontal differential heating can be much higher than the molecular level when flow fluctuations exist. Moreover, the Rayleigh number for world oceans is about $10^{20}$ times larger than in our experiments. It is speculated that the flow will no longer be simply steady and 
laminar, and it should be some type of eddying flow in the regime of an extremely high Rayleigh number (Paparella \& Young 2002).

Results discussed in this study were obtained from a single container, with a fixed horizontal length, with the exception of two special runs. The strength of the steady circulation confirms the 1/5-power law of Rossby (1965) in terms of the Rayleigh number based on the horizontal length. We have found some evidence of unsteady states. Additionally, we cannot rule out the possibility of multiple states under the same thermal forcing condition.

More importantly, it is unclear what will happen if the eddying flow exists instead of laminar flow; if a tank with a larger dimension is used in similar experiments, what power law would the circulation obey. Thus, the question whether or not the Atlantic Ocean under pure thermal forcing from the sea surface can develop a sizable overturning circulation remains a great challenge for enquiring minds.

W. W. was supported by The National Natural Science Foundation of China through grant 40476010 and the Research Fund for the Doctoral Program of Higher Education through grant 20030423011. R. X. H. was supported by the National Science Foundation through grant OCE-0094807 and the National AeroSpace Administration through Contract 1229833 (NRA-00-OES-05) to the Woods Hole Oceanographic Institution. Reviewers' comments helped with the presentation. Woods Hole Contribution 11342.

\section{Appendix. Scaling of the circulation}

Discussion here follows the analysis of Paparella \& Young (2002). Assuming flow is two-dimensional, the non-Boussinesq equations governing the flow are conservation of mass, momentum, and thermal energy (White 1974), plus the equation of state

$$
\begin{gathered}
\frac{\partial \rho}{\partial t}+\nabla \cdot(\rho \boldsymbol{u})=0, \\
\rho \frac{\mathrm{D} \boldsymbol{u}}{\mathrm{Dt}}=-\nabla p+\rho \boldsymbol{g}+\mu \nabla^{2} \boldsymbol{u}, \\
\rho c_{p} \frac{\mathrm{D} T}{\mathrm{D} t}=\kappa \rho c_{p} \nabla^{2} T-p \nabla \cdot \boldsymbol{u}+\Phi, \\
\rho=\rho_{0}\left[1-\alpha\left(T-T_{0}\right)\right],
\end{gathered}
$$

where $\rho\left(\rho_{0}\right)$ is the density (mean density) of the fluid, $g$ is the acceleration due to gravity, $T$ is temperature, $T_{0}$ is a constant reference temperature, $p$ pressure, $c_{p}$ the specific heat, $\alpha$ is the thermal expansion coefficient, and $\Phi$ the dissipation function

$$
\Phi=\mu\left[2\left(\frac{\partial u}{\partial x}\right)^{2}+2\left(\frac{\partial w}{\partial z}\right)^{2}+\left(\frac{\partial w}{\partial x}+\frac{\partial u}{\partial z}\right)^{2}\right]+\lambda\left(\frac{\partial u}{\partial x}+\frac{\partial w}{\partial z}\right)^{2},
$$

where $\lambda=-2 \mu / 3$ is commonly accepted as a parameter for most fluids.

Taking the dot product of (A2) with the velocity vector leads to the conservation equation of mechanical energy

$$
\frac{\partial}{\partial t}\left[\rho E_{k}+\rho g z\right]+\nabla \cdot\left[\left(\rho E_{k}+\rho g z+p\right) \boldsymbol{u}\right]=p \nabla \cdot \boldsymbol{u}+\mu \nabla^{2} E_{k}+\mu\|\nabla \boldsymbol{u}\|^{2}
$$

where $E_{k}=\frac{1}{2} \boldsymbol{u} \cdot \boldsymbol{u}$ is the kinetic energy per unit mass and $\|\nabla \boldsymbol{u}\|^{2} \equiv \nabla u \cdot \nabla u+\nabla w \cdot \nabla w$ is the deformation of velocity. 
While in a steady state, no mechanical energy enters/leaves the system through the boundaries, averaging (A 6) over the volume leads to a simple balance between the pressure work and dissipation

$$
\langle p \nabla \cdot \boldsymbol{u}\rangle-\mu\left\langle\|\nabla \boldsymbol{u}\|^{2}\right\rangle=0
$$

where \langle\rangle denotes the ensemble average over the volume of the circulation cell. This definition is used here as there is no motion above (or below for case 2) the circulation cell.

Assuming $\alpha=$ const, and $p=p_{0}+p^{\prime}, p^{\prime}=-\rho_{0} g z$, to a very good approximation, (A 3 ) is reduced to a balance between the first two terms

$$
\nabla \cdot \boldsymbol{u}=-\frac{1}{\rho_{0}} \frac{\mathrm{D} \rho}{\mathrm{D} t}=-\alpha \frac{\mathrm{D} T}{\mathrm{D} t}=-\alpha \kappa \nabla^{2} T
$$

thus,

$$
\langle p \nabla \cdot \boldsymbol{u}\rangle=-\rho_{0} g \alpha \kappa\left\langle z \nabla^{2} T\right\rangle .
$$

Using the Green formulae, $\left\langle z \nabla^{2} T\right\rangle$ is reduced to

$$
\left\langle z \nabla^{2} T\right\rangle=\frac{1}{D L} \iint z\left(\frac{\partial^{2} T}{\partial x^{2}}+\frac{\partial^{2} T}{\partial z^{2}}\right) \mathrm{d} x \mathrm{~d} z=-\frac{\bar{T}_{t o p}-\bar{T}_{b o t t o m}}{D},
$$

where $D$ is the depth of the water, $\bar{T}_{\text {top }}$ and $\bar{T}_{\text {bottom }}$ are the mean temperature at the top and bottom boundary, respectively. The mean temperature at the upper boundary cannot be higher than $T_{h}$, i.e. $\bar{T}_{\text {top }} \leqslant T_{h}$, and $T_{\text {bottom }}=\left(T_{h}+T_{c}\right) / 2$; thus, (A 10) is reduced to

$$
\left\langle z \nabla^{2} T\right\rangle \leqslant-\frac{\Delta T}{2 D}
$$

and the mean mechanical energy generation rate is

$$
\frac{1}{\rho_{0}}\langle p \nabla \cdot \boldsymbol{u}\rangle \leqslant \frac{\kappa \alpha g \Delta T}{2 D}=\frac{\kappa g^{\prime}}{2 D} .
$$

This relation provides an upper limit for the mechanical energy convertible from thermal energy: $\left(1 / \rho_{0}\right)\langle p \nabla \cdot \boldsymbol{u}\rangle D L \leqslant\left(\kappa g^{\prime} / 2\right) L$, which is independent of the water depth. Since in partial-penetrating circulation flow, both the mechanical energy generation and dissipation occur within the cell, the mean mechanical energy generation rate within the cell is

$$
\frac{1}{\rho}\langle p \nabla \cdot \boldsymbol{u}\rangle_{c e l l}=\frac{1}{h_{c} L} \frac{\kappa g^{\prime}}{2} L=\frac{\kappa g^{\prime}}{2 h_{c}} .
$$

From (A 7) the mean dissipation rate within the cell should be

$$
v\left\langle\|\nabla \boldsymbol{u}\|^{2}\right\rangle_{\text {cell }}=\frac{\kappa g^{\prime}}{2 h_{c}},
$$

where $h_{c}$ is the cell height of the circulation.

Equation (A 14) is just the result of Paparella \& Young (2002). For flow with fluctuations, the left-hand side of (A 14) represents the total dissipation rate, $\varepsilon_{\text {total }}=v\left\langle\|\nabla \boldsymbol{u}\|^{2}\right\rangle_{\text {cell }}$, which is composed of two items: $\varepsilon_{m}$ and $\varepsilon_{t}$. $\varepsilon_{m}$ is related to the mean flow and can be represented as

$$
\varepsilon_{m}=v\left\langle\|\nabla \overline{\boldsymbol{u}}\|^{2}\right\rangle_{c e l l}
$$

and $\varepsilon_{t}$ represent the dissipation rate related to velocity fluctuations. 
It is well-known that the dissipation induced by velocity fluctuations is essentially three-dimensional. Since our measurements give two-dimensional velocity only, we have to estimate such a dissipation rate in the following way. Applying the method that was used in analysing turbulent flow, and assuming that the fluctuation is isotropic, the dissipation rate, $\varepsilon_{t}$, can be calculated as follows (Tennekes \& Lumley 1972; Hinze 1975; Melville, Veron \& White 2002)

$$
\varepsilon_{t}=2 v\left\langle S_{i j} S_{i j}\right\rangle, \quad S_{i j}=\frac{1}{2}\left(\frac{\partial u_{i}{ }^{\prime}}{\partial x_{j}}+\frac{\partial u_{j}{ }^{\prime}}{\partial x_{i}}\right), \quad \varepsilon_{t}=15 v\left\langle\left(\frac{\partial u^{\prime}}{\partial x}\right)^{2}\right\rangle_{\text {cell }},
$$

where primes denote the fluctuation of the velocity. Note that flows observed in our experiments are not turbulent flow, and they are far from being isotropic; thus, the results obtained from this calculation should be interpreted as the rough estimated upper bounds.

A direct application of the Paparella \& Young (2002) formulae provides an upper limit for the mechanical energy convertible from surface thermal forcing in the world ocean. Assume the equator-pole temperature difference is $\Delta T \simeq 30 \mathrm{~K}$, the mean depth of the world ocean is $\bar{D} \simeq 3.75 \mathrm{~km}, \alpha \simeq 2 \times 10^{-4} \mathrm{~K}^{-1}, \kappa \simeq 1.5 \times 10^{-7} \mathrm{~m}^{2} \mathrm{~s}^{-1}$, so $\langle p \nabla \cdot \boldsymbol{u}\rangle \sim \rho_{0} g \alpha \kappa \Delta T / 2 \bar{D} \simeq 1.2 \times 10^{-9} \mathrm{Wm}^{-3}$. The total volume of the ocean is $1.324 \times 10^{18} \mathrm{~m}^{3}$, so the total conversion rate is about $1.5 \times 10^{9} \mathrm{~W}$. This is 1000 times smaller than the rate of tidal dissipation. Since the total amount of heat flux going through the oceans is about $2 \times 10^{15} \mathrm{~W}$, the efficiency of the ocean as a heat engine is about $7 \times 10^{-7}$.

\section{REFERENCES}

Beardsley, R. C. \& Festa, J. F. 1972 A numerical model of convection driven by a surface stress and non-uniform horizontal heating. J. Phys. Oceanogr. 2, 444-455.

Defant, A. 1961 Physical Oceanography, vol. 1. Pergamon.

Hinze, J. O. 1975 Turbulence. McGraw-Hill.

Huang, R. X. 1999 Mixing and energetics of the thermohaline circulation. J. Phys. Oceanogr. 29, $727-746$.

HuAng, R. X. 2004 Ocean, energy flow in. Encyclopedia of Energy (ed. C. J. Cleveland), vol. 4, pp. 497-509. Elsevier.

JEFFREYS, H. 1925 On the motions produced by differences of temperature and humidity. $Q . J . R$. Met. Soc. 51, 347-356.

LIDE, D. R. (Ed.) 2001 Handbook of Chemistry and Physics. CRC Press.

Melville, W. K., Veron, F. \& White, C. J. 2002 The velocity field under breaking waves: coherent structures and turbulence. J. Fluid Mech. 454, 203-233.

Miller, R. C. 1968 A thermally convecting fluid heated non-uniformly from below. PhD thesis, MIT.

Mullarney, J. C., Griffiths, R. W. \& Hughes, G. O. 2004 Convection driven by differential heating at a horizontal boundary. J. Fluid. Mech. 516, 181-209.

Munk, W. H. \& Wunsch, C. 1998 The Moon and mixing: abyssal recipes II. Deep-Sea Res. I 45, $1977-2010$.

Paparella, F. \& Young, W. R. 2002 Horizontal convection is non turbulent. J. Fluid Mech. 466, 205-214.

Rossby, T. 1965 On thermal convection driven by non-uniform heating from below; an experimental study. Deep-Sea Res. 12, 9-16.

Rossby, T. 1998 Numerical experiments with a fluid heated non-uniformly from below. Tellus 50A, 242-257.

Sandström, J. W. 1908 Dynamicsche Versuche mit Meerwasser. Annln Hydrograph. Martimen Met. 36, 6-23. 
SANDSTRÖM, J. W. 1916 Meteorologische Studien im schwedischen Hochgebirge. Goteborgs K. Vetenskaps-och Vitterhetssamhalles Handl. Ser. 4, 22(2), 48pp.

Siedler, G. \& Peters, H. 1986 Properties of sea water. In Numerical Data and Functional Relationships in Science and Techonology, New Series, vol. 3a, Oceanography (ed. J. Sundermann), pp. 233-264. Springer.

Siggers, J. H., Kerswell, R. R. \& Balmforth, N. J. 2004 Bounds on horizontal convection. J. Fluid. Mech. 517, 55-70.

Somerville, R. C. 1967 A non-linear spectral model of convection in a fluid unevenly heated from below. J. Atmos. Sci. 24, 665-676.

Stern, M. E. 1975 Ocean Circulation Physics. Academic.

Tennekes, H. \& Lumley, J. L. 1972 A First Course in Turbulence. MIT Press.

White, F. M. 1974 Viscous Fluid Flow, 2nd edn. McGraw-Hill.

Wunsch, C. \& Ferrari, R. 2004: Vertical mixing, energy, and the general circulation of the oceans. Annu. Rev. Fluid Mech. 36, 281-314.

XIA, K. Q., Sun, C. \& ZHOU, S. Q. 2003 Particle image velocimetry measurement of the velocity field in turbulent thermal convection. Phys. Rev. E 68, 066303. 